TRANSACTIONS OF THE

AMERICAN MATHEMATICAL SOCIETY

Volume 360, Number 10, October 2008, Pages 5121-5143

S 0002-9947(08)04483-8

Article electronically published on April 10, 2008

\title{
PBW-BASES OF COIDEAL SUBALGEBRAS AND A FREENESS THEOREM
}

\author{
V. K. KHARCHENKO
}

\begin{abstract}
Let $H$ be a character Hopf algebra. Every right coideal subalgebra $\mathbf{U}$ that contains the coradical has a PBW-basis which can be extended up to a PBW-basis of $H$. If additionally $\mathbf{U}$ is a bosonization of an invariant with respect to the left adjoint action subalgebra, then $H$ is a free left (and right) $\mathbf{U}$-module with a free PBW-basis over $\mathbf{U}$. These results remain valid if $H$ is a braided Hopf algebra generated by a categorically ordered subset of primitive elements. If the ground field is algebraically closed, the results are still true provided that $H$ is a pointed Hopf algebra with commutative coradical and is generated over the coradical by a direct sum of finite-dimensional YetterDrinfeld submodules of skew primitive elements.
\end{abstract}

\section{INTRODUCTION}

A recent survey by G. Letzter [17] offers a panorama of the use of one-sided coideal subalgebras in studying Drinfeld-Jimbo quantum enveloping algebras. The very one-sided comodule subalgebras, but not the Hopf subalgebras, turn out to be the Galois objects in the Galois theory for Hopf algebra actions (see a recent survey by T.Yanai 45 and the references there). In particular the Galois correspondence theorem for the actions on a free algebra set up a one-to-one correspondence between all right coideal subalgebras and all intermediate free subalgebras; see V.O. Ferreira, L.S.I. Murakami, and A. Paques 7.

In the present paper we consider right coideal subalgebras in character Hopf algebras, the Hopf algebras generated by skew-primitive semi-invariants. This class includes the quantum enveloping algebras of Kac-Moody algebras, all their generalizations (see S.-J. Kang [11, G. Benkart, S.-J. Kang, and D. Melville 2, M. Costantini and M. Varagnolo [4, J. Towber [42]), the bosonizations of quantum symmetric (bitensor, external, Nichols) algebras related to diagonal braidings, and so on. To some extent this class of Hopf algebras can be treated as the abstractly defined class of all quantum universal enveloping algebras (see [13, Theorem 6.1], [14]). We prove the following general statement on the structure of the right coideal subalgebras.

Theorem 1.1. Let $H$ be a character Hopf algebra. Every right coideal subalgebra that contains all group-like elements has a PBW-basis which can be extended up to a PBW-basis of $H$.

Received by the editors February 8, 2006.

2000 Mathematics Subject Classification. Primary 16W30, 16W35; Secondary 17B37.

Key words and phrases. Hopf algebra, coideal subalgebra, PBW-basis.

The author was supported by PAPIIT IN 108306-3, UNAM, México.

(C)2008 American Mathematical Society Reverts to public domain 28 years from publication 
There are many publications on the construction of a PBW-basis for Hopf algebras. M. Rosso [33] and, independently of him, H. Yamane [44] have constructed the PBW-basis for Drinfeld-Jimbo algebras of type $A_{n}$. Then G. Lusztig in his fundamental works [19, 20] has found the PBW-bases for arbitrary Drinfeld-Jimbo and Lusztig quantum enveloping algebras. These bases and their modifications have been considered in a number of ensuing papers: J. Towber [42, F. Gavarini 9, V. Chari and N. Xi 3, M. Reineke 31], B. Leclerc [16. Let us mention an original approach based on the Ringel-Hall algebras [5, 6, 32.

The author in 12 by means of a combinatorial method based on the LyndonShirshov standard words has shown that every character Hopf algebra does have a PBW-basis. This statement has an equivalent braided version: Every primitively generated braided Hopf algebra with a diagonal braiding has a PBW-basis. The most general result to date in this direction is due to $\mathrm{S}$. Ufer [43, who has found a large class of braided Hopf algebras where the method applies.

There exist several investigations dealing with the freeness of a Hopf algebra $H$ over a right coideal subalgebra U. M. Takeuchi showed that $H$ (and more generally each right (H, $\mathbf{U}$ )-Hopf module) is a free $\mathbf{U}$-module provided that $\mathbf{U}$ is contained in the center of $H$ and satisfies some additional restrictions: see [39, p. 453]. In particular if $H$ is a commutative pointed Hopf algebra, there is a 1-1 correspondence between right coideal subalgebras over which $H$ is free, and Hopf ideals of $H$. A. Masuoka 21] proved that a finite-dimensional Hopf algebra $H$ is left and right free over a right coideal subalgebra $\mathbf{U}$ if and only if $\mathbf{U}$ is a Frobenius algebra, while S. Skryabin [38] has proved that any right coideal subalgebra indeed is Frobenius. As an application of Theorem 1.1 we prove the following freeness theorem.

Theorem 1.2. If in Theorem 1.1 the right coideal subalgebra $\mathbf{U}$ is a bosonization of an invariant under the left adjoint action subalgebra, then $H$ has a free PBW-basis as a left and right $\mathbf{U}$-module.

The ad-invariant one-sided coideal subalgebras are the key constituents of the locally finite part of quantum enveloping algebras [17, p. 139]. Constants of coordinate differential calculi provide more examples of ad-invariant one-sided coideal subalgebras [15, Corollary 4.3, Lemma 4.13].

If the ground field is algebraically closed, Theorem 1.1 and Theorem 1.2 remain valid in a more general setting when $H$ is a pointed Hopf algebra with commutative coradical and is generated over the coradical by a direct sum of finite-dimensional Yetter-Drinfeld submodules of skew primitive elements (Theorem 6.1, Corollary 6.3). Any character Hopf algebra in these terms is generated by one-dimensional YD-submodules.

The results have an equivalent formulation in terms of braided Hopf algebras (Corollary 17.1) that admit a generalization as follows. Suppose that a braided space $(V, \tau)$ has a completely ordered basis $X$. For each $x \in X$ we denote by $V_{\leq x}$ a subspace spanned by all $y \leq x$. The basis $X$ is categorically ordered if all such spaces are categorical:

$$
\tau\left(V_{\leq x} \otimes V_{\leq y}\right) \subseteq V_{\leq y} \otimes V_{\leq x} .
$$

Theorem 1.3. Every right coideal subalgebra $\mathbf{U}$ of a braided Hopf algebra $R$ generated by a braided subspace $V$ of primitive elements with categorically ordered basis has a set of $P B W$ generators that can be extended up to a set of $P B W$ generators of $R$. If $\mathbf{U}$ is ad-invariant, then $R$ has a free left and right $P B W$-basis over $\mathbf{U}$. 
Theorem 1.1 is new for Hopf subalgebras too, while the freeness theorem for pointed Hopf algebras over Hopf subalgebras is the fundamental result of D. Radford [27. In this case just the existence of a free PBW-basis provides some new information. It should be remembered that there has been considerable interest in the problem of determining the Hopf subalgebras over which $H$ is free as a module. We mention the famous theorem of Nichols and Zoeller 23] that every finite-dimensional Hopf algebra is a free left (and right) module over each Hopf subalgebra. A braided version is due to B. Scharfschwerdt [34] (see also M. Takeuchi 40). In the infinite-dimensional case, it has been known for a long time that a Hopf algebra need not be free over a Hopf subalgebra (U. Olberst and H.-J. Schneider [26], M. Takeuchi [39, Section 5], D. Radford [29]), although it is true in various special cases when the Hopf subalgebra is still supposed to be finite-dimensional (D. Radford [28, W.D. Nichols and M.B. Richmond [25], H.-J. Schneider [35], M. Graña [10]), or has other types of restrictions (D. Radford [27, M. Takeuchi [39], W.D. Nichols and M.B. Zoeller [24]).

Finally we remark that in this paper we do not require $H$ to be finitely generated; this even slightly generalizes [12.

\section{Preliminaries}

2.1. PBW-generators. Let $S$ be an algebra over a field $\mathbf{k}$ and let $A$ be a subspace of $S$ with a basis $\left\{a_{j} \mid j \in J\right\}$. A linearly ordered subset $V \subseteq S$ is said to be a set of $P B W$-generators of $S$ over $A$ if there exists a function $h: V \rightarrow \mathbf{Z}^{+} \cup\{\infty\}$, called the height function, such that the set of all products

$$
a_{j} v_{1}^{n_{1}} v_{2}^{n_{2}} \cdots v_{k}^{n_{k}}
$$

where $j \in J, \quad v_{1}<v_{2}<\ldots<v_{k} \in V, \quad n_{i}<h\left(v_{i}\right), 1 \leq i \leq k$ is a basis of $S$. The value $h(v)$ is referred to as the height of $v$ in $V$. If $A=\mathbf{k}$ is the ground field, then we shall call $V$ simply a set of $P B W$-generators of $S$.

Definition 2.1. Let $V$ be a set of PBW-generators of $S$ over a subalgebra $A$. Suppose that the set of all words in $V$ as a free monoid has its own order $\prec$ (that is, $a \prec b$ implies $c a d \prec c b d$ for all words $a, b, c, d$ in $V$ ).

1) A leading word of $s \in S$ is the maximal word $m=v_{1}^{n_{1}} v_{2}^{n_{2}} \cdots v_{k}^{n_{k}}$ that appears in the decomposition of $s$ in the basis (2.1).

2) A leading term of $s$ is the sum am of all terms $\alpha_{i} a_{i} m$ that appear in the decomposition of $s$ in the basis (2.1), where $m$ is the leading word of $s$.

3) The order $\prec$ is compatible with the $P B W$-decomposition related to $V$ if the leading word of each product $W=w_{1} w_{2} \cdots w_{m}, w_{i} \in V \cup\left\{a_{j}\right\}$ (considered as an element of $S$ ) is less than or equal to the word $W^{t}$ that appears from $w_{1} w_{2} \cdots w_{m}$ by deletion of all letters $a_{j}$.

2.2. Associated graded algebra. Let $\Gamma$ be a completely ordered additive (commutative) monoid. A $\Gamma$-filtration on an algebra $S$ is a map $D$ from $S$ to $\Gamma$ extended by a symbol $-\infty$ with the properties

$$
\begin{array}{lll}
\text { 1. } & D(x)=-\infty \Longleftrightarrow x=0, & D\left(1 \cdot \mathbf{k}^{*}\right)=0, \\
\text { 2. } & D(x-y) \leq \max \{D(x), D(y)\}, & D(x y) \leq D(x)+D(y) .
\end{array}
$$

The element $D(x)$ is the degree of $x$. We denote by $S_{\gamma}$ the subspace of all elements of degree $\leq \gamma$, while by $S_{\gamma}^{-}$we denote the subspace of all elements of degree $<\gamma$. 
Example 2.2 (Filtration by constitution). Suppose that an algebra $S$ is generated over a subalgebra $A$ by a set $X$. That is, there exists a homomorphism $\xi: A\langle X\rangle \rightarrow S$, where $A\langle X\rangle$ is the free product $A * \mathbf{k}\langle X\rangle$. A constitution of a word $u$ in $A \cup X$ is a family of nonnegative integers $\left\{m_{x}, x \in X\right\}$ such that $u$ has $m_{x}$ occurrences of $x$. Certainly almost all $m_{x}$ in the constitution are zero. We fix an arbitrary complete order, $<$, on the set $X$.

Let $\Gamma$ be the free additive (commutative) monoid generated by $X$. The monoid $\Gamma$ is a completely ordered monoid with respect to the following order:

$$
m_{1} x_{i_{1}}+m_{2} x_{i_{2}}+\ldots+m_{k} x_{i_{k}}>m_{1}^{\prime} x_{i_{1}}+m_{2}^{\prime} x_{i_{2}}+\ldots+m_{k}^{\prime} x_{i_{k}}
$$

if the first from the left nonzero number in $\left(m_{1}-m_{1}^{\prime}, m_{2}-m_{2}^{\prime}, \ldots, m_{k}-m_{k}^{\prime}\right)$ is positive, where $x_{i_{1}}>x_{i_{2}}>\ldots>x_{i_{k}}$ in $X$. We associate a formal degree $D(u)=\sum_{x \in X} m_{x} x \in \Gamma$ to a word $u$ in $A \cup X$, where $\left\{m_{x} \mid x \in X\right\}$ is the constitution of $u$ (in [8, $\S 2.1$ ] the formal sum $D(u)$ is called the weight of $u$ ). Respectively, if $f=\sum \alpha_{i} u_{i} \in A\langle X\rangle, 0 \neq \alpha_{i} \in \mathbf{k}$, then

$$
D(f)=\max _{i}\left\{D\left(u_{i}\right)\right\} .
$$

On $S$ we define a $\Gamma$-filtration related to $\xi: A * \mathbf{k}\langle X\rangle \rightarrow S$ as follows:

$$
D(s)=\min \{D(f) \mid \xi(f)=s\} .
$$

With every $\Gamma$-filtered algebra $S$ a $\Gamma$-graded algebra is associated in the obvious way. For each $\gamma \in \Gamma$, write $\operatorname{gr}_{\gamma} S$ for the linear space $S_{\gamma} / S_{\gamma}^{-}$. Then

$$
\operatorname{gr} S=\bigoplus_{\gamma \in \Gamma} \operatorname{gr}_{\gamma} S,
$$

with the product defined by

$$
\left(x+S_{\gamma}^{-}\right)\left(y+S_{\delta}^{-}\right)=x y+S_{\gamma+\delta}^{-} .
$$

If the filtration is as in Example 2.2 then the algebra gr $S$ is generated by gr $X \cong X$ and $\operatorname{gr} A=A$.

Lemma 2.3. If the associated graded algebra gr $S$ for a $\Gamma$-filtered algebra $S$ has a set of homogeneous $P B W$-generators, $V=\left\{v_{i}\right\}$, over a subspace $A \subseteq S_{0}$, then each set of representatives $\hat{v}_{i}, v_{i}=\hat{v}_{i}+S_{D\left(v_{i}\right)}^{-}$, is a set of $P B W$-generators of $S$ over $A$.

This lemma has been proved by S. Ufer [43, Proposition 46] for the case $\Gamma=\mathbf{Z}^{+}$. In the general case the proof is quite similar.

Lemma 2.4. Let $\mathbf{U}$ be a subalgebra of a $\Gamma$-filtered algebra $S$. If $\operatorname{gr} S$ is a free left (right) $\Gamma$-graded module over gr $\mathbf{U}$, then $S$ is a free left (right) module over $\mathbf{U}$.

This is part of the folklore.

2.3. Thin elements and replacement of basis. Suppose that an algebra $S$ has a set $V$ of PBW-generators over a subalgebra $A$, and $1 \in\left\{a_{j}\right\}$. Let $\prec$ be any complete order of the free monoid of words in $V$ which is compatible with the PBW-decomposition related to $V$. An element $c \in S$ is said to be a thin element if its decomposition in the basis (2.1) has the from

$$
c=v^{m}+\sum \alpha_{i j} a_{j} W_{i},
$$

where $W_{i} \prec v^{m}$, and either $m$ divides the height of $v$ in $V$, or $h(v)=\infty$. 
Let $T \subseteq S$ be some set of thin elements. Suppose that for each $v \in V$ there exists at most one element $c_{v} \in T$ with the leading term of the form $v^{m}, m \geq 1$. One may construct a new set of PBW-generators, $P_{T}$, related to $T$ in the following way.

If in $T$ there does not exist an element with the leading term of the form $v^{m}$, $m \geq 1$, then we include $v$ in $P_{T}$ and define the height $h_{T}(v)$ related to $T$ to be equal to $h(v)$.

If there exists an element $c_{v} \in T$ with the leading term $v^{m}$ and $m>1$, then we include in $P_{T}$ both elements: $v$ and $c_{v}$. In this case we define the height of $v$ in $P_{T}$ to be equal to $m$, while the height of $c_{v}$ related to $P_{T}$ is the quotient $h(v) / m$.

If there exists an element $c_{v} \in T$ with the leading term $v$ (that is, if $m=1$ ), then we include in $P_{T}$ just $c_{v}$ (that accidentally may be equal to $v$ ). In this case the height of $c_{v}$ with respect to $T$ by definition equals $h(v)$.

We extend on $P_{T}$ the order $<$ in the natural way: $c_{v}<w$ if and only if $(v \leq w$ $\& c_{v} \neq w$ ); and $c_{v}<c_{w}$ if and only if $v<w$. In particular $c_{v}<v$, provided that $m>1$, where $m$ is defined by $c_{v}$ in (2.5).

Lemma 2.5. The set $P_{T}$ is a set of $P B W$-generators of $S$ over $A$.

Proof. We have to prove that the monotonous restricted words (2.1) with $v_{i} \in P_{T}$ are linearly independent in $S$, and they span $S$.

By definition if $\theta_{1}<\theta_{2} \in P_{T}, \theta_{1}=v_{1}^{m_{1}}+\cdots, \theta_{2}=v_{2}^{m_{2}}+\cdots$, then either $v_{1}<v_{2}$, or $v_{1}=v_{2}=v$ with $\theta_{1}=c_{v}=v^{m}+\cdots, \theta_{2}=v$. Therefore each monotonous word in $P_{T}$ has a form

$$
\theta_{1}^{n_{1}} v_{1}^{r_{1}} \theta_{2}^{n_{2}} v_{2}^{r_{2}} \cdots \theta_{k}^{n_{k}} v_{k}^{r_{k}},
$$

where $v_{1}<v_{2}<\cdots<v_{k}, \theta_{i}=c_{v_{i}}=v_{i}^{m_{i}}+\cdots$. Of course if $m_{i}=1$, then $r_{i}=0$, while if $m_{i}>1$, then $r_{i} \geq 0$. The word (2.6) is restricted if and only if $n_{i}<h_{T}\left(\theta_{i}\right)=h\left(v_{i}\right)$ in the case $m_{i}=1$, and $n_{i}<h_{T}\left(\theta_{i}\right)=h\left(v_{i}\right) / m_{i}, r_{i}<m_{i}$ otherwise. If we replace $\theta_{i}:=v_{i}^{m_{i}}+\cdots$ in (2.6) and then develop multiplication, we get a linear combination $W+\sum_{q} \alpha_{q} W_{q}$ of words in $V \cup\left\{a_{j}\right\}$, where

$$
W=v_{1}^{n_{1} m_{1}+r_{1}} v_{2}^{n_{1} m_{2}+r_{2}} \cdots v_{k}^{n_{k} m_{k}+r_{k}} .
$$

Let us as above denote by $W^{t}$ a word in $V$ that appears from $W$ by deletion of all $a_{j}$. Since $\prec$ is a monoidal order, we have $W_{q}^{t} \prec W$, for all $q$. If $m_{i}>1$, then

$$
n_{i} m_{i}+r_{i} \leq\left(\frac{h\left(v_{i}\right)}{m_{i}}-1\right) m_{i}+\left(m_{i}-1\right)=h\left(v_{i}\right)-1 \text {. }
$$

If $m_{i}=1$, again $n_{i} m_{i}+r_{i}=n_{i}<h\left(v_{i}\right)$. Hence (2.7) is a monotonous restricted word, provided that so is (2.6). Since the order $\prec$ is compatible with the PBW-decomposition related to $V$, all words $v_{1} v_{2} \cdots v_{k}$ that appears in the PBWdecomposition (2.1) of $\sum_{q} \alpha_{q} W_{q}$ are less than (2.7). It is important to note that, in this way, to different monotonous restricted words in $P_{T}$ correspond different monotonous restricted words in $V$.

Suppose that a linear combination, $\Xi=\sum \alpha_{i j} a_{j} U_{i}$, over $A$ of monotonous restricted words in $P_{T}$ equals zero in $S$. Let us first in $\Xi$ replace each $\theta \in P_{T}$ by its representation, $\theta:=v^{m}+\cdots$, in terms of PBW-generators $V$, and next develop the multiplication. We get a linear combination $\Xi^{\prime}=\sum \beta_{k} W_{k}$ of words in $P \cup\left\{a_{j} \mid j \in J\right\}$. Since $\prec$ is a monoidal order $(a \prec b$ implies $c a d \prec c b d)$, the maximal word $W_{0}$ among $W_{k}^{t}$ appears in the decomposition of summands $a_{j} U$ with the 
only word $U$. If $U$ has form (2.6), then $W_{0}$ has form (2.7). Since the order $\prec$ is compatible with the PBW-decomposition related to $V$, the maximal word of $\Xi^{\prime}$ in the PBW-decomposition appears just in summands $\alpha_{s j} a_{j} W_{0}$, where the equality $U_{s}=U$ defines the index $s$. Hence $\Xi^{\prime} \neq 0$ in $S$, a contradiction.

To see that monotonous restricted words in $P_{T} \cup\left\{a_{j}\right\}$ span $S$ we may use a standard induction on words ordered by $\prec$. Indeed, for any monotonous restricted word in $V$,

$$
W=v_{1}^{s_{1}} v_{2}^{s_{2}} \cdots v_{k}^{s_{k}},
$$

we have $s_{i}=n_{i} m_{i}+r_{i}, r_{i}<m_{i}, n_{i}<h_{T}\left(\theta_{i}\right)$, where either $\theta_{i}=c_{v_{i}}=v_{i}^{m_{i}}+\cdots \in$ $T$, or the set $T$ has no elements with the leading word $v_{i}^{m}, m \geq 1$ (and hence $\left.\theta_{i}=v_{i} \in P_{T}, m_{i}=1\right)$. Suppose by induction that the values of all super-words smaller than $W$ belong to the linear space spanned by monotonous restricted words in $P_{T} \cup\left\{a_{j}\right\}$. The difference between $W$ and (2.6) is a linear combination of words $U$ in $P_{T} \cup\left\{a_{j}\right\}$ such that $U^{t}$ are less than $W$. Due to the comparability of $\prec$ with the PBW-decomposition related to $V$, the $\mathrm{PBW}$-decomposition of any such word has only summands $\alpha a_{j} W^{\prime}$ with $W^{\prime} \prec W$. By induction the lemma is proved.

Remark. In the proof we have seen that the leading term of (2.6) in basis (2.1) equals (2.7).

\section{PBW-Basis of a CHARACTER Hopf Algebra}

Recall that a Hopf algebra $H$ is referred to as a character Hopf algebra if the group $G$ of all grouplike elements is commutative and $H$ is generated over $\mathbf{k}[G]$ by skew primitive semi-invariants $s_{i}, i \in I$ :

$$
\Delta\left(s_{i}\right)=s_{i} \otimes 1+g_{s_{i}} \otimes s_{i}, \quad g^{-1} s_{i} g=\chi^{s_{i}}(g) s_{i}, \quad g, g_{s_{i}} \in G,
$$

where $\chi^{s_{i}}, i \in I$ are characters of the group $G$. Let us associate a variable $x_{i}$ to $s_{i}$. For each word $u$ in $X=\left\{x_{i} \mid i \in I\right\}$ we denote by $g_{u}$ an element of $G$ that appears from $u$ by replacing each $x_{i}$ with $g_{s_{i}}$. In the same way we denote by $\chi^{u}$ a character that appears from $u$ by replacing of each $x_{i}$ with $\chi^{s_{i}}$. We define a bilinear skew commutator by the formula

$$
[u, v]=u v-p_{u v} v u
$$

where by definition $p_{u v}=\chi^{u}\left(g_{v}\right)=p(u, v)$.

The group $G$ acts on the free algebra $\mathbf{k}\langle X\rangle$ by $g^{-1} u g=\chi^{u}(g) u$, where $u$ is an arbitrary monomial in $X$. The skew group algebra $G\langle X\rangle$ has the natural Hopf algebra structure

$$
\Delta\left(x_{i}\right)=x_{i} \otimes 1+g_{s_{i}} \otimes x_{i}, \quad i \in I, \quad \Delta(g)=g \otimes g, g \in G .
$$

The construction of the PBW-basis given in 12 requires $I$ to be finite. In order to get a PBW-basis in the general case one may slightly modify this construction as follows.

We fix a Hopf algebra homomorphism $\xi: G\langle X\rangle \rightarrow H, \xi\left(x_{i}\right)=s_{i}, \xi(g)=g, i \in I$, $g \in G$, and consider the filtration $D$ related to $\xi$ as defined in Example 2.2. This filtration is compatible with the Hopf algebra structure, that is,

$$
\Delta\left(H_{\gamma}\right) \subseteq \sum_{\delta+\varepsilon=\gamma} H_{\delta} \otimes H_{\varepsilon}, \sigma\left(H_{\gamma}\right) \subseteq H_{\gamma},
$$


where $\sigma$ is the antipode. Therefore gr $H$ is also a character Hopf algebra generated by $\mathbf{k}[G]$ and $\xi(X)$.

By Lemmas 2.3 and 2.4 in what follows we may suppose that $H$ is a $\Gamma$-graded character Hopf algebra (or, in other words, it is homogeneous in each of the generators $s_{i}$ ).

On the set of all words in $X$ we fix the lexicographical order with the priority from the left to the right, where a proper beginning of a word is considered to be greater than the word itself. This order is not monoidal: we have $x>x^{2}$, while $x y<x^{2} y$ if $y<x$. It has neither ACC, nor DCC: $x>x^{2}>\ldots>x^{n}>\ldots$; $x y<x^{2} y<\ldots<x^{n} y<\ldots$. For by these reasons we need one more order, the Hall (or deg-lex) one, $u \prec v$ if $D(u)<D(v)$ or $D(u)=D(v) \& u<v$. Since there exists just a finite number of words in $X$ of a given constitution, the Hall order indeed is a complete order of the free monoid.

A nonempty word $u$ is called a standard word (or Lyndon word, or LyndonShirshov word) if $v w>w v$ for each decomposition $u=v w$ with nonempty $v, w$. A nonassociative word is a word where brackets [,] are somehow arranged to show how multiplication applies. If $[u]$ denotes a nonassociative word, then by $u$ we denote an associative word obtained from $[u]$ by removing the brackets (of course $[u]$ is not uniquely defined by $u$ in general).

The set of standard nonassociative words is the biggest set $S L$ that contains all variables $x_{i}$ and satisfies the following properties.

1) If $[u]=[[v][w]] \in S L$, then $[v],[w] \in S L$, and $v>w$ are standard.

2) If $[u]=\left[\left[\left[v_{1}\right]\left[v_{2}\right]\right][w]\right] \in S L$, then $v_{2} \leq w$.

Every standard word has the only alignment of brackets such that the appeared nonassociative word is standard (the Shirshov theorem [36]). In order to find this alignment one may use the following procedure: The factors $v, w$ of the nonassociative decomposition $[u]=[[v][w]]$ are the standard words such that $u=v w$ and $v$ has the minimal length ([37, see also [18]).

Definition 3.1. A super-letter is a polynomial that equals a nonassociative standard word where the brackets mean (3.2). A super-word is a word in super-letters.

By Shirshov's theorem every standard word $u$ defines the only super-letter; in what follows we will denote it by $[u]$. The order on the super-letters is defined in the natural way: $[u]>[v] \Longleftrightarrow u>v$. We should stress that this order is not complete: If $x>y$, there are infinite chains of super-letters

$$
[x y]>\left[x y^{2}\right]>\ldots>\left[x y^{n}\right]>\ldots ;[x y]<\left[x^{2} y\right]<\ldots<\left[x^{n} y\right]<\ldots .
$$

Nevertheless the Hall order on super-words is still a complete monoidal order.

Definition 3.2. A super-letter $[u]$ is called hard in $H$ provided that its value in $H$ is not a linear combination of values of super-words in smaller than $[u]$ super-letters.

Definition 3.3. We say that a height of a hard in $H$ super-letter $[u]$ equals $h=$ $h([u])$ if $h$ is the smallest number such that: first, $p_{u u}$ is a primitive $t$-th root of unity and either $h=t$ or $h=t l^{r}$, where $l=\operatorname{char}(\mathbf{k})$; and then the value in $H$ of $[u]^{h}$ is a linear combination of super-words in less than $[u]$ super-letters. If there exists no such number, then the height equals infinity.

Theorem 3.4. The values of all hard in $H$ super-letters form a set of $P B W$ generators of $H$ over $\mathbf{k}[G]$. 
Proof. If $X$ is a finite set, this statement has been proved in [12, Theorem 2]; see a footnote on page 267. In the general case the algebra $G\langle X\rangle$ has a covering by $G\left\langle X_{\alpha}\right\rangle$, where $X_{\alpha}$ runs through all finite subsets of $X$. We shall consider each of $X_{\alpha}$ as an ordered subset of $X$, and $G\left\langle X_{\alpha}\right\rangle$ as a $\Gamma$-graded subalgebra of $G\langle X\rangle$. Respectively $H$ has a covering by the finitely generated $\Gamma$-graded character Hopf subalgebras $H_{\alpha} \stackrel{d f}{=} \xi\left(G\left\langle X_{\alpha}\right\rangle\right)$.

By Definition 3.2 every hard in $H$ super-letter $[u]$ is hard in $H_{\alpha}$ as soon as $u \in G\left\langle X_{\alpha}\right\rangle$. If a super-letter $[v] \in G\left\langle X_{\alpha}\right\rangle$ is not hard in $H$, then by definition $\xi([v])=\xi(A)$, where $A$ is a linear combination of super-words in less than $[v]$ super-letters. Since $H$ is $\Gamma$-graded, all super-words of the sum $A$ have the same constitution as the word $v$ does. In particular all super-words of $A$ belong to $G\left\langle X_{\alpha}\right\rangle$. Hence $[v]$ is not hard in $H_{\alpha}$.

In the same way the height function is independent of $\alpha$.

The following statements provide important properties of the PBW-basis defined by the hard super-letters.

Lemma 3.5 ([12, Lemma 8]). The coproduct of a super-letter $[w]$ has a representation

$$
\Delta([w])=[w] \otimes 1+g_{w} \otimes[w]+\sum_{i} \alpha_{i} g\left(W_{i}^{\prime \prime}\right) W_{i}^{\prime} \otimes W_{i}^{\prime \prime},
$$

where $W_{i}^{\prime}$ are nonempty words in less than $[w]$ super-letters.

Lemma 3.6. The Hall order of the super-words is compatible with the $P B W$ decomposition related to the hard super-letters (see Definition 2.1).

Proof. Let $W$ be a super-word. There exists the following natural diminishing process of the decomposition. First, in $\mathbf{k}\langle X\rangle$ according to [12, Lemma 7] we decompose the super-word $W$ in a linear combination of smaller monotonous super-words. Then, we replace each nonhard super-letter with the decomposition of its value in $H$ that exists by Definition 3.2 , and again decompose the appeared super-words in linear combinations of smaller monotonous super-words, and so on, until we get a linear combination of monotonous super-words in hard super-letters. If they are not restricted, we may apply Definition 3.3 and repeat the process until we get only monotonous restricted words in hard super-letters.

Lemma 3.7. If $[w]$ is a super-letter, then

$$
\Delta\left(\xi\left([w]^{m}\right)\right)=\sum_{j=0}^{m}\left[\begin{array}{l}
m \\
j
\end{array}\right]_{q} g_{w}^{m-j} \xi([w])^{j} \otimes \xi([w])^{m-j}+\sum_{i} \alpha_{i} g\left(V_{i}\right) \xi\left(U_{i}\right) \otimes \xi\left(V_{i}\right),
$$

where $\left[\begin{array}{l}m \\ j\end{array}\right]_{q}$ are the $q$-binomial coefficients with $q=p(w, w)$, and $U_{i}$ are basis superwords such that the first from the left super-letter is less than $[w]$.

Proof. The $m$-th power of the right-hand side of (3.4) after developing the product takes the form (3.5), where each of the $U_{i}$ is a product of $m$ super-words some of which are equal to $[w]$ (but not all of them!) and others are equal to the $W_{i}^{\prime}$ 's. Hence $U_{i}$ as a super-word is less than $[w]^{m}$ with respect to the lexicographical ordering of words in super-letters. Let us decompose $\xi\left(U_{i}\right)$ in the PBW-basis defined by the hard super-letters. According to Lemma 3.6 we turn to the formula (3.5) where 
still $U_{i}<[w]^{m}$. This implies the required property since the first super-letter in a basis super-word is always the minimal one.

\section{Right Coideal Subalgebras}

Theorem 4.1. Let $H$ be a character Hopf algebra. Every right coideal subalgebra $\mathbf{U} \supseteq \mathbf{k}[G]$ has a $P B W$-basis that can be extended up to a PBW-basis of $H$.

By Lemma 2.3 it suffices to show that gr $\mathbf{U}$ as a subalgebra of $\operatorname{gr} H$ has a required basis. Since grU is a right coideal subalgebra of $\operatorname{gr} H$, we may still suppose that $H$ is a $\Gamma$-graded character Hopf algebra and $\mathbf{U}$ is a $\Gamma$-graded coideal subalgebra.

Lemma 4.2. Let $w \in \mathbf{U}$. If $\pi, \mu: H \rightarrow H$ are linear maps such that $(\pi \otimes \mu) \Delta(w)=$ $a \otimes b$ with $b \neq 0$, then $a=\pi(c)$ for some $c \in \mathbf{U}$.

Proof. We have $(\pi \otimes \mu) \Delta(\mathbf{U}) \subseteq \pi(\mathbf{U}) \otimes H$. Hence $a \otimes b \in \pi(\mathbf{U}) \otimes H$, and $a \in$ $\pi(\mathbf{U})$.

We shall use this evident statement as a basic tool. Note that once we have a PBW-basis of $H$, to define a linear map it suffices to fix its values on the restricted monotonous words in an arbitrary way.

Let $[u]$ be a hard super-letter. Suppose that in $\mathbf{U}$ there exists an element $c$ with the leading super-word $[u]^{m}, m \geq 1$. Since $G \subseteq \mathbf{U}$, we may suppose that the super-word $[u]^{m}$ appears one time with the trivial coefficient:

$$
c=\xi\left([u]^{m}+\sum_{j} \alpha_{j} g_{j}[u]^{m}+\sum_{i} \alpha_{i} g_{i} W_{i} R_{i}\right)
$$

where $W_{i}$ are nonempty basis super-words in less than $[u]$ super-letters, while the $R_{i}$ are basis super-words in greater than or equal to $[u]$ super-letters, $\alpha_{i}, \alpha_{j} \in$ $\mathbf{k}, g_{i}, g_{j} \in G, g_{j} \neq 1$.

Denote by $\iota$ the natural projection $H \rightarrow \mathbf{k}, \iota(g \xi(W))=0$, unless $g=1, W=\emptyset$. Since $\Delta(g W)=(g \otimes g) \Delta(W)$, we have

$$
(\mathrm{id} \otimes \iota)(\Delta(c))=\xi\left([u]^{m}+\sum_{g_{i}=1} \alpha_{i} W_{i} R_{i}\right) \otimes 1 .
$$

Thus, by Lemma 4.2 ,

$$
c^{\prime}=\xi\left([u]^{m}+\sum_{g_{i}=1} \alpha_{i} W_{i} R_{i}\right) \in \mathbf{U}
$$

In what follows we fix the notation $c_{u}$ for one of the elements from $\mathbf{U}$ of the form (4.2) that has the minimal possible $m$.

Lemma 4.3. In the representation (4.2) of the chosen element $c_{u}$ either $m=1$, or $p_{u u}$ is a primitive $t$-th root of unity and $m=t$ or (in the case of positive characteristic) $m=t(\operatorname{char} \mathbf{k})^{s}$. In particular $c_{u}$ is a thin element; see (2.5).

Proof. If $m=1$ there is nothing to prove. Let $m>1$. For each $k, 1 \leq k<m$ we consider the following linear map set up on the PBW-basis of super-words:

$$
\pi_{k}(g \xi(W))=\left\{\begin{array}{cl}
0, & \text { if } W \prec[u]^{k}, \\
g \xi(W), & \text { otherwise. }
\end{array}\right.
$$

By means of formula (3.5) we have

$$
\left(\pi_{k} \otimes \pi_{m-k}\right) \Delta\left(\xi([u])^{m}\right)=\left[\begin{array}{l}
m \\
k
\end{array}\right] g_{u}^{m-k} \xi([u])^{k} \otimes \xi([u])^{m-k} .
$$


By Lemma 3.5 and Lemma 3.6 the coproduct $\Delta\left(\xi\left(W_{i}\right)\right)$ is the sum of tensors $g \xi\left(W_{i}^{\prime}\right) \otimes \xi\left(W_{i}^{\prime \prime}\right)$, where all the $W_{i}^{\prime}$ are the basis super-words lexicographically smaller than $[u]^{m}$ with the only exception being the one that equals $g\left(W_{i}\right) \otimes$ $\xi\left(W_{i}\right)$. Hence $\left(\pi_{k} \otimes \pi_{m-k}\right) \Delta\left(\xi\left(W_{i} R_{i}\right)\right)$ is a sum of tensors of the form $g \pi_{k}\left(\xi\left(R_{i}^{\prime}\right)\right) \otimes$ $\pi_{m-k}\left(\xi\left(W_{i} R_{i}^{\prime \prime}\right)\right)$. Again by Lemma 3.6 we have $\pi_{m-k}\left(\xi\left(W_{i} R_{i}^{\prime \prime}\right)\right)=0$. Hence

$$
\left(\pi_{k} \otimes \pi_{m-k}\right) \Delta\left(\xi\left(W_{i} R_{i}\right)\right)=0 .
$$

Thus we may write

$$
\left(\pi_{k} \otimes \pi_{m-k}\right) \Delta\left(c_{u}\right)=\left[\begin{array}{l}
m \\
k
\end{array}\right]_{q} g_{u}^{m-k} \xi([u])^{k} \otimes \xi([u])^{m-k} .
$$

If $\left[\begin{array}{l}m \\ k\end{array}\right] \neq 0$, then by Lemma 4.2 we find $c \in \mathbf{U}$ such that $\pi_{k}(c)=g_{u}^{m-k} \xi\left([u]^{k}\right)$. By definition of $\pi_{k}$ this equality means that the PBW-decomposition of $g_{u}^{k-m} c$ has the form (4.2) with $k$ in place of $m$. Since by the choice of $c_{u}$ the number $m$ is minimal, we get $\left[\begin{array}{l}m \\ k\end{array}\right]_{q}=0,1 \leq k<m$. This system of equations implies $q^{m}=1$ and either $m$ equals the multiplicative order $t$ of $q$ or $m=t(\operatorname{char} \mathbf{k})^{s}$.

By Lemma 2.5 the set $T$ of all the above defined elements $c_{u}$ has an extension up to a set $P_{T}$ of PBW-generators of $H$ over $\mathbf{k}[G]$. Now Theorem 4.1 follows from the proposition below.

Proposition 4.4. An element $c \in H$ belongs to $\mathbf{U}$ if and only if all $P B W$ generators in the $P B W$-decomposition of $c$ with respect to $P_{T}$ belong to $T$. In particular $T$ is a set of $P B W$-generators of $\mathbf{U}$ over $\mathbf{k}[G]$.

To prove this statement we will need some additional properties of the PBWbasis defined by $P_{T}$. We extend the order "<" already defined on $P_{T}$ onto the set of all words in $P_{T}$ as the lexicographical order. The order " $\prec$ " is the Hall order induced by the degree function $D$; that is, $W \prec U$ if and only if either $D(W)<D(U)$, or $D(W)=D(U) \& W<U$. We have to stress that the order on the set of letters $P_{T}$ differs from the order on the set of one-letter words $P_{T}$.

We start with connections between these two PBW-decompositions.

Lemma 4.5. The leading term of a monotonous restricted word in $P_{T}$,

$$
\theta_{1}^{n_{1}}\left[u_{1}\right]^{r_{1}} \theta_{2}^{n_{2}}\left[u_{2}\right]^{r_{2}} \cdots \theta_{k}^{n_{k}}\left[u_{k}\right]^{r_{k}},
$$

under the $P B W$-decomposition related to the hard super-letters equals

$$
\left[u_{1}\right]^{n_{1} m_{1}+r_{1}}\left[u_{2}\right]^{n_{1} m_{2}+r_{2}} \cdots\left[u_{k}\right]^{n_{k} m_{k}+r_{k}}
$$

where $\theta_{i}=\left[u_{i}\right]^{m_{i}}+\cdots$. Conversely if

$$
W=\left[u_{1}\right]^{s_{1}}\left[u_{2}\right]^{s_{2}} \cdots\left[u_{k}\right]^{s_{k}}
$$

is a monotonous restricted super-word in hard super-letters, then its leading term in the decomposition with respect to $P_{T}$ equals (4.7), where $n_{i}=\left\lfloor s_{i} / m_{i}\right\rfloor, r_{i}=$ $s_{i}-n_{i} m_{i}$.

Proof. The first part of the lemma has been proved in Lemma 2.5. The second part follows by induction on super-words ordered by the Hall order. Indeed the difference $E$ between $W$ and (4.7) is a linear combination of super-words that are less than $W$. By Lemma 3.6 all basis super-words in the PBW-decomposition of $E$ are less than $W$. It remains to note that if $W^{\prime} \prec W$ is another basis super-word, then the word (4.7) related to $W^{\prime}$ is less than that related to $W$. 
Lemma 4.6. The Hall order on the words in $P_{T}$ is compatible with the $P B W$ decomposition related to $P_{T}$ (see Definition 2.1).

Proof. Let $W$ be a word in $P_{T}$. The word $W$ has the form (4.7), where $\theta_{i}=c_{u_{i}}=$ $\xi\left(\left[u_{i}\right]^{m_{i}}+\cdots\right)$, while the $u_{i}$ 's are not necessarily increasing from the left to the right. If we replace $\theta_{i}:=\left[u_{i}\right]^{m_{i}}+\cdots$ in (4.7) and then develop the multiplication in $G\langle X\rangle$, we get a linear combination $\Sigma$ over $\mathbf{k}[G]$ of super-words with the leading term

$$
\left[u_{1}\right]^{n_{1} m_{1}+r_{1}}\left[u_{2}\right]^{n_{1} m_{2}+r_{2}} \cdots\left[u_{k}\right]^{n_{k} m_{k}+r_{k}} .
$$

By Lemma 3.6 each super-word $W_{1}$ in the PBW-decomposition of $\xi(\Sigma)$ is less than or equal to (4.10). That is, if $W_{1}$ is different from (4.10), we may write

$$
W_{1}=\left[u_{1}\right]^{n_{1} m_{1}+r_{1}}\left[u_{2}\right]^{n_{2} m_{2}+r_{2}} \cdots\left[u_{s}\right]^{n_{s} m_{s}+r_{s}}\left[u_{s+1}\right]^{t}[v] \cdots ;
$$

here $[v]$ is the first from the left super-letter where $W_{1}$ differs from (4.10), and $0 \leq t<n_{s+1} m_{s+1}+r_{s+1}$. Since $W_{1}$ is monotonous, we have $v>u_{s+1}$, provided that $t \neq 0$. However in this case $W_{1}$ is greater than (4.10). Hence $t=0$, and $u_{s+1}>v \geq u_{s} \geq u_{s-1} \geq \ldots \geq u_{1}$.

If all inequalities among the $u_{i}$ 's in the above chain are strict, then according to Lemma 4.5, all words of the PBW-decomposition with respect to $P_{T}$ of $\xi\left(W_{1}\right)$ are less than or equal to

$$
\theta_{1}^{n_{1}}\left[u_{1}\right]^{r_{1}} \theta_{2}^{n_{2}}\left[u_{2}\right]^{r_{2}} \cdots \theta_{s}^{n_{s}}\left[u_{s}\right]^{r_{s}} \theta_{v},
$$

where $\theta_{v}=c_{v}$, or $\theta_{v}=[v]$. Hence they are less than $W$.

If not all inequalities are strict, say $u_{1}<u_{2}<\ldots<u_{p}=\cdots=u_{q}<u_{q+1}$, $q \leq s$, then again by Lemma 4.5 the $P_{T}$-leading word of $\xi\left(W_{1}\right)$ starts with

$$
\theta_{1}^{n_{1}}\left[u_{1}\right]^{r_{1}} \cdots \theta_{p-1}^{n_{p-1}}\left[u_{p-1}\right]^{r_{p-1}} \theta_{p}^{\left(n_{p}+\cdots+n_{q}+n_{0}\right) m_{p}}\left[u_{p}\right]^{r_{0}},
$$

where $r_{p}+\cdots+r_{q}=n_{0} m_{p}+r_{0}, 0 \leq r_{0}<m_{p}$. It is still less than $W$ since by definition $\theta_{p}<\left[u_{p}\right]$, provided that $m_{p}>1$.

Lemma 4.7. The coproduct of each $\theta \in P_{T}$ has a representation

$$
\Delta(\theta)=\theta \otimes 1+g_{\theta} \otimes \theta+\sum_{i} \alpha_{i} g_{i} W_{i}^{\prime} \otimes W_{i}^{\prime \prime},
$$

where $W_{i}^{\prime}, W_{i}^{\prime \prime}$ are restricted monotonous words (products) in $P_{T}$, and for every $i$ either $W_{i}^{\prime}$ or $W_{i}^{\prime \prime}$ starts with a letter that is less than $\theta$.

Proof. Let $\theta=\xi\left([u]^{m}+\sum_{i} W_{i} R_{i}\right)$ be the decomposition of $\theta \in P_{T}$ with respect to the hard super-letters. By Lemma 3.7 and Lemma 4.3, we have

$$
\Delta\left(\xi\left([u]^{m}\right)\right)-\xi\left([u]^{m}\right) \otimes 1-g_{u}^{m} \otimes \xi\left([u]^{m}\right)=\sum_{i} \alpha_{i} g_{i} \xi\left(U_{i}\right) \otimes \xi\left(V_{i}\right),
$$

where the $U_{i}$ are basis super-words starting with smaller than $[u]$ super-letters. By the second part of Lemma 4.5 each summand of the decomposition of $\xi\left(U_{i}\right)$ in the PBW-basis defined by $P_{T}$ starts by $c_{w}$ or $[w]$ with $w<u$.

Let $W_{i}=[w] U$, where $[w]<[u]$. By Lemma 3.5 we have

$$
\Delta\left(W_{i} R_{i}\right)=\sum_{j}\left(V_{i j}^{\prime} \otimes V_{i j}^{\prime \prime}\right) \Delta\left(U R_{i}\right)+\left(g_{w} \otimes[w]\right) \Delta\left(U R_{i}\right)
$$


where all $V_{i j}^{\prime}$ are nonempty super-words in less than $[u]$ super-letters. All left hand sides of the tensors $\left(V_{i j}^{\prime} \otimes V_{i j}^{\prime \prime}\right) \Delta\left(U R_{i}\right)$ start with smaller than $[u]$ super-letters. Hence by Lemma 3.6 it remains to use the converse part of Lemma 4.5 .

In perfect analogy, if $\Delta\left(U R_{i}\right)=\sum_{j} U_{i j}^{\prime} \otimes U_{i j}^{\prime \prime}$, then $\left(g_{w} \otimes[w]\right) \Delta\left(U R_{i}\right)=g_{w} U_{i j}^{\prime} \otimes$ $[w] U_{i j}^{\prime \prime} R_{i}$. Therefore the right hand side of each tensor that appears in the $P_{T^{-}}$ decomposition of $\left(g_{w} \otimes \xi([w])\right) \Delta\left(\xi\left(U R_{i}\right)\right)$ starts with a letter that is less than $\theta$.

Lemma 4.8. Let $\theta \in P_{T}$. The coproduct of $\theta^{n}$ has a decomposition

$$
\Delta\left(\theta^{n}\right)=\sum_{j=0}^{n}\left[\begin{array}{l}
n \\
j
\end{array}\right]_{q} g_{\theta}^{n-j} \theta^{j} \otimes \theta^{n-j}+\sum_{i} \alpha_{i} g_{i} W_{i}^{\prime} \otimes W_{i}^{\prime \prime},
$$

where $\left[\begin{array}{l}n \\ j\end{array}\right]_{q}$ are $q$-binomial coefficients with $q=p(\theta, \theta), W_{i}^{\prime}, W_{i}^{\prime \prime}$ are restricted monotonous words in $P_{T}$, and for every $i$ either $W_{i}^{\prime}$ or $W_{i}^{\prime \prime}$ starts with a letter that is less $\operatorname{than} \theta$.

Proof. If we develop multiplication in the $n$-th power of the right hand side of (4.14), then we get the first sum of (4.15) and a $\mathbf{k}[G]$-linear combination of tensors $\theta^{i} W^{\prime} \otimes \theta^{j} W^{\prime \prime}$, where $i$ and $j$ may be zero, but either $W^{\prime}$ or $W^{\prime \prime}$ starts with a less than $\theta$ letter. Let it be $W^{\prime}$. By Lemma 4.6 the PBW-decomposition of $\theta^{i} W^{\prime}$ has only words that are less than $\theta^{i} W^{\prime}$ in lexicographical order. Since these words are monotonous, no one of them may start with a letter that is greater than or equal to $\theta$. The lemma is proved.

Proof of Proposition 4.4. Let to the contrary $c \in \mathbf{U}$ be an element of the minimal degree whose decomposition in the $P_{T}$-basis has super-letters $[u] \in P_{T} \backslash T$. Since $\mathbf{U}$ is a subalgebra, we may suppose that each term of the decomposition has such a super-letter.

Let $U=\theta_{1}^{n_{1}} \theta_{2}^{n_{2}} \cdots \theta_{k}^{n_{k}}, \theta_{1}<\theta_{2}<\cdots<\theta_{k}$ be the leading word of $c$ in the $P_{T}$-basis. Since $G \subseteq \mathbf{U}$, we may suppose that $U$ appears one time with the trivial coefficient:

$$
c=U+\sum_{j} \alpha_{j} g_{j} U+\sum_{i} \beta_{i} g_{i} U_{i}
$$

where $D\left(U_{i}\right)=D(U), \alpha_{j}, \beta_{i} \in \mathbf{k}, g_{i}, g_{j} \in G, g_{j} \neq 1$.

Denote by $\iota$ the natural projection $H \rightarrow \mathbf{k}, \iota(g W)=0$, unless $g=1, W=\emptyset$. Here $W$ is an arbitrary restricted monotonous word in $P_{T}$. Since $\Delta(g W)=(g \otimes g) \Delta(W)$, we have

$$
(\mathrm{id} \otimes \iota)(\Delta(c))=\left(U+\sum_{g_{i}=1} \beta_{i} U_{i}\right) \otimes 1 .
$$

Thus, by Lemma 4.2 ,

$$
c^{\prime}=U+\sum_{g_{i}=1} \beta_{i} U_{i} \in \mathbf{U}
$$

where $U_{i}<U$. To get a contradiction we consider two cases.

Case 1. Suppose that $\theta_{k} \notin T$; that is, $\theta_{k}=[u]$ and in $\mathbf{U}$ there does not exist an element with the leading super-word $[u]^{m}, 1 \leq m \leq n_{k}$. Let us define the following 
two linear maps set up on the PBW-basis related to $P_{T}$ :

$$
\begin{gathered}
\pi(g W)=\left\{\begin{array}{cl}
0, & \text { if } W \prec \theta_{k}^{n_{k}} ; \\
g W, & \text { otherwise; }
\end{array}\right. \\
\nu(g W)=\left\{\begin{array}{cl}
0, & \text { if } W \prec \theta_{1}^{n_{1}} \cdots \theta_{k-1}^{n_{k-1}} ; \\
g W, & \text { otherwise. }
\end{array}\right.
\end{gathered}
$$

Let us show first that

$$
(\pi \otimes \nu)(\Delta(U))=g \theta_{k}^{n_{k}} \otimes \theta_{1}^{n_{1}} \cdots \theta_{k-1}^{n_{k-1}}, \quad g \in G .
$$

Since the word $U$ is monotonous, by Lemma 4.8 we have

$$
\Delta\left(\theta_{i}^{n_{i}}\right)=\sum_{j_{i}} \alpha_{j_{i}} g_{j_{i}} W_{j_{i}}^{\prime} \otimes W_{j_{i}}^{\prime \prime}, \quad i<k,
$$

where for each $j_{i}, i \leq k$, either $W_{j_{i}}^{\prime}$ starts by a letter from $P_{T}$ that is less than $\theta_{k}$, or $W_{j_{i}}^{\prime \prime}$ starts by a letter from $P_{T}$ that is less than $\theta_{i}$, with the only exception being $W_{j_{i}}^{\prime}=\emptyset, W_{j_{i}}^{\prime \prime}=\theta_{i}^{n_{i}}$. In the same way,

$$
\Delta\left(\theta_{k}^{n_{k}}\right)=\sum_{j_{k}} \alpha_{j_{k}} g_{j_{k}} W_{j_{k}}^{\prime} \otimes W_{j_{k}}^{\prime \prime},
$$

where either $W_{j_{k}}^{\prime}$ or $W_{j_{i}}^{\prime \prime}$ starts by a letter from $P_{T}$ that is less than $\theta_{k}$, with exceptions of the form $W_{j_{k}}^{\prime}=\theta_{k}^{j}, W_{j_{k}}^{\prime \prime}=\theta_{k}^{n_{k}-j}$.

$\Delta(U)$ is a right linear combination over $\mathbf{k}[G]$ of tensors

$$
E=W_{j_{1}}^{\prime} W_{j_{2}}^{\prime} \cdots W_{j_{k}}^{\prime} \otimes W_{j_{1}}^{\prime \prime} W_{j_{2}}^{\prime \prime} \cdots W_{j_{k}}^{\prime \prime}
$$

Let $W_{j_{s}}^{\prime}$ be the first from the left nonempty factor in $E$.

If, first, $s \neq k$, then the tensor takes the form

$$
E=W_{j_{s}}^{\prime} \cdots W_{j_{k}}^{\prime} \otimes \theta_{1}^{n_{1}} \cdots \theta_{s-1}^{n_{s-1}} W_{j_{s}}^{\prime \prime} \cdots W_{j_{k}}^{\prime \prime} .
$$

If $W_{j_{s}}^{\prime}$ starts by a less than $\theta_{k}$ letter, then the left hand side of $E$ is less than $\theta_{k}^{n_{k}}$; hence by Lemma 4.6 we get $(\pi \otimes \nu)(E)=0$.

If $W_{j_{s}}^{\prime \prime}$ starts by a less than $\theta_{s}$ letter, then the right hand side is less than $\theta_{1}^{n_{1}} \cdots \theta_{k-1}^{n_{k-1}}$, and again $(\pi \otimes \nu)(E)=0$.

If, next, $s=k$, then the tensor has the form

$$
E=W_{j_{k}}^{\prime} \otimes \theta_{1}^{n_{1}} \cdots \theta_{k-1}^{n_{k-1}} W_{j_{k}}^{\prime \prime} .
$$

Since $D\left(W_{j_{k}}^{\prime}\right)=n_{k} D\left(\theta_{k}\right)-D\left(W_{j_{k}}^{\prime \prime}\right)<n_{k} D\left(\theta_{k}\right)$, unless $W_{j_{k}}^{\prime \prime}=\emptyset$, we get $\pi\left(W_{j_{k}}^{\prime}\right)=0$ with the only exception being $W_{j_{k}}^{\prime}=\theta_{k}^{n_{k}}$. Thus $\pi \otimes \nu$ kills all tensors in the PBWdecomposition of $\Delta(U)$ except for one of them. This proves (4.20).

Let us show, further, that

$$
(\pi \otimes \nu)\left(U_{i}\right)=0 .
$$

Since $U_{i}<U$, we have $U_{i}=\theta_{1}^{n_{1}} \cdots \theta_{s}^{n_{s}} \theta_{s+1}^{r} \eta \cdots$, where $0 \leq r<n_{s+1}, \eta<\theta_{s+1}$. If $r \neq 0$, then $\theta_{s+1}<\eta$ since the word $U_{i}$ is monotonous. Thus $r_{s}=0$; that is,

$$
U_{i}=\theta_{1}^{n_{1}} \cdots \theta_{s}^{n_{s}} \eta \cdots, \quad \eta<\theta_{s+1} .
$$

By means of Lemma 4.7 we have

$$
\Delta(\eta)=\sum_{j} \alpha_{j} g_{j} V_{j}^{\prime} \otimes V_{j}^{\prime \prime},
$$


where for each $j$, either $V_{j}^{\prime}$ or $V_{j}^{\prime \prime}$ starts by a letter from $P_{T}$ which is less than $\theta_{s+1}$. Thus $\Delta\left(U_{i}\right)$ is a right linear combination over $\mathbf{k}[G]$ of tensors

$$
E=W_{j_{1}}^{\prime} \cdots W_{j_{s}}^{\prime} V_{j}^{\prime} \cdots \otimes W_{j_{1}}^{\prime \prime} \cdots W_{j_{s}}^{\prime \prime} V_{j}^{\prime \prime} \cdots
$$

If $W_{j_{1}}^{\prime}=\cdots=W_{j_{s}}^{\prime}=V_{j}^{\prime}=\emptyset$, then the right hand side takes the form $\theta_{1}^{n_{1}} \cdots \theta_{s}^{n_{s}} \eta \cdots$, which is less than $\theta_{1}^{n_{1}} \cdots \theta_{k-1}^{n_{k-1}}$; hence $(\pi \otimes \nu)(E)=0$.

Let $W_{j_{r}}^{\prime}, r \leq s$ be the first from the left nonempty word in $E$. The tensor takes the form

$$
E=W_{j_{r}}^{\prime} \cdots W_{j_{s}}^{\prime} V_{j}^{\prime} \cdots \otimes \theta_{1}^{n_{1}} \cdots \theta_{r-1}^{n_{r-1}} W_{j_{r}}^{\prime \prime} \cdots W_{j_{s}}^{\prime \prime} V_{j}^{\prime \prime} \cdots
$$

Since either $W_{j_{r}}^{\prime}$ starts with a less than $\theta_{k}$ letter, or $W_{j_{r}}^{\prime \prime}$ starts with a less than $\theta_{r}$ letter, again $(\pi \otimes \nu)(E)=0$.

If the first from the left nonempty factor is $V_{j}^{\prime}$, then in the same way either $V_{j}^{\prime}$ starts with a letter $\leq \eta<\theta_{s+1} \leq \theta_{k}$, or $V_{j}^{\prime \prime}$ does. In both cases $(\pi \otimes \nu)(E)=0$.

Thus $(\pi \otimes \nu)\left(\Delta\left(U_{i}\right)\right)=0$. Now taking into account (4.17), we get

$$
(\pi \otimes \nu)\left(\Delta\left(c^{\prime}\right)\right)=g \theta_{k}^{n_{k}} \otimes \theta_{1}^{n_{1}} \cdots \theta_{k-1}^{n_{k-1}}, g \in G .
$$

Lemma 4.2 implies that in $\mathbf{U}$ there exists an element $c^{\prime \prime}$ such that $\pi\left(c^{\prime \prime}\right)=$ $g \theta_{k}^{n_{k}}$. Definition (4.18) of $\pi$ shows that $\theta_{k}^{n_{k}}=[u]^{n_{k}}$ is the leading word of $c^{\prime \prime}$ in the $P_{T}$-decomposition. By Lemma 4.5 the leading super-word of $c^{\prime \prime}$ equals $[u]^{n_{k}}$. According to the construction of $T$, there exists an element $c_{u} \in T$ of the form $c_{u}=[u]^{m}+\cdots, m \leq n_{k}$. This contradicts the conditions of the first case $\left([u]=\theta_{k} \notin T, h_{T}([u])>n_{k}\right)$.

Case 2. Suppose that $\theta_{k} \in T$. In this case $\theta_{k} \in \mathbf{U}$; therefore one of the letters $\theta_{1}, \ldots, \theta_{k-1}$ does not belong to $T$. By the inductive supposition no one element with the leading word $\theta_{1}^{n_{1}} \cdots \theta_{k-1}^{n_{k-1}}$ belongs to $\mathbf{U}$. At the same time, in perfect analogy with the first case (up to left-right symmetry in the consideration of tensors), we have

$$
(\nu \otimes \pi)\left(\Delta\left(c^{\prime}\right)\right)=g \theta_{1}^{n_{1}} \cdots \theta_{k-1}^{n_{k-1}} \otimes \theta_{k}^{n_{k}} .
$$

Hence by Lemma 4.2 there exists $c^{\prime \prime} \in \mathbf{U}$ such that $\nu\left(c^{\prime \prime}\right)=g \theta_{1}^{n_{1}} \cdots \theta_{k-1}^{n_{k-1}}$. Definition (4.19) of $\nu$ implies that the leading word of $c^{\prime \prime}$ indeed equals $\theta_{1}^{n_{1}} \cdots \theta_{k-1}^{n_{k-1}}$, a contradiction. Proposition 4.4 and Theorem 4.1 are completely proved.

Corollary 4.9. If a right coideal subalgebra $\mathbf{U}$ of a character Hopf algebra $H$ contains $\mathbf{k}[G]$, then it is a free left $\mathbf{k}[G]$-module, and

$$
\mathbf{k}[G] H={ }_{\mathbf{k}[G]} \mathbf{U} \otimes_{\mathbf{k}} H^{+},
$$

where $H^{+}$is the space spanned by all monotonous restricted words in $P_{T} \backslash T$.

Proof. The definition of PBW-generators over $\mathbf{k}[G]$ implies that $\mathbf{U}$ is a free left $\mathbf{k}[G]$-module generated by all monotonous restricted words $W$ in $T$. Denote by $W^{+}$ the word that appears from $W$ by cancellation of all letters from $T$, while by $W^{-}$the word that appears from $W$ by cancellation of all letters from $P_{T} \backslash T$. Both words $W^{+}$and $W^{-}$are monotonous and restricted. Hence the map $W \rightarrow W^{-} \otimes W^{+}$ defines the required decomposition. 


\section{5. $\mathrm{AD}_{l}$-INVARIANT SUBALGEBRAS}

We are reminded that the (left) adjoint action of a Hopf algebra $H$ on itself is given by $\left(\operatorname{ad}_{l} h\right)(\theta)=\sum h_{(1)} \theta S\left(h_{(2)}\right)$, where $S$ is the antipode, $\Delta(h)=\sum h_{(1)} \otimes h_{(2)}$.

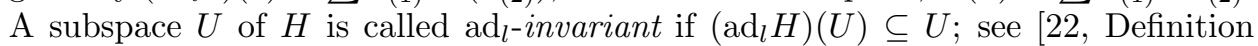
3.4.1]. If the ideal generated by $s_{i}, i \in I$ has zero intersection with $\mathbf{k}[G]$, then $\pi: s_{i} \rightarrow 0, \pi: g \rightarrow g$ defines a Hopf algebra projection $\pi: H \rightarrow \mathbf{k}[G]$. In this case by the Radford theorem, see [1, $\S \S 1.5,1.7], H$ has a decomposition in a biproduct $H=A \# \mathbf{k}[G]$ by means of the isomorphism $h \rightarrow \vartheta\left(h_{(1)}\right) \# \pi\left(h_{(2)}\right)$ with $\vartheta(h)=\sum h_{(1)} \pi\left(S h_{(2)}\right)$, where $A=\vartheta(H)$ is a subalgebra generated by $s_{i}, i \in I$. If $\mathbf{U}$ is a right coideal subalgebra of $H$, and $\mathbf{k}[G] \subseteq \mathbf{U}$, then $U \stackrel{d f}{=} \vartheta(\mathbf{U}) \subseteq \mathbf{U}$. Hence $\mathbf{U}$ is a bosonization of $U$; that is, $\mathbf{U}=U \# \mathbf{k}[G]$.

Theorem 5.1. If a right coideal subalgebra $\mathbf{U}$ of a character Hopf algebra $H$ is a bosonization of an $\operatorname{ad}_{l}$-invariant subalgebra $U$, then $H$ has a $P B W$-basis over $\mathbf{U}$, and it is a left and right free $\mathbf{U}$-module.

In what follows we keep the notation of Section 3 and Section 4. In particular we regard $H$ as a $\Gamma$-filtered Hopf algebra. The $\operatorname{map} \operatorname{ad}_{l} h$ satisfies $\operatorname{ad}_{l} h\left(H_{\gamma}\right) \subseteq$ $H_{\gamma+D(h)}$. Hence $\operatorname{ad}_{l} \bar{h} \stackrel{d f}{=} \operatorname{ad}_{l}\left(h+H_{D(h)}^{-}\right)$is a homogeneous map of degree $D(h)$; that is, $\operatorname{ad}_{l} \bar{h}: \operatorname{gr}_{\gamma} H \rightarrow \operatorname{gr}_{\gamma+D(h)} H$, and $\left(\operatorname{ad}_{l} \bar{h}\right)\left(\operatorname{gr}_{\gamma} \mathbf{U}\right) \subseteq \operatorname{gr}_{\gamma+D(h)} \mathbf{U}$. In particular $\operatorname{gr} \mathbf{U}$ is an $\operatorname{ad}_{l}$-invariant right coideal $\Gamma$-graded subalgebra of $\operatorname{gr} H$. From this point on we shall assume that $H=\operatorname{gr} H, \mathbf{U}=\operatorname{gr} \mathbf{U}$ are $\Gamma$-graded objects (see Lemma 2.3 and Lemma 2.4), while the chosen elements $\theta \in T$ belong to $U$.

Recall that $W^{+}$is the word that appears from $W$ by cancellation of all letters from $T$, and $W^{-}$is the word that appears from $W$ by cancellation of all letters from $P_{T} \backslash T$. If $W_{1}$ is a monotonous restricted word in $T$ and $W_{2}$ is a monotonous restricted word in $P_{T} \backslash T$, then there exists the only monotonous restricted word $W$ in $P_{T}$ such that $W^{-}=W_{1}, W^{+}=W_{2}$. We denote this word by $W_{1} \circ W_{2}$.

We shall define a new degree function $D^{+}$on the set of all words in $P_{T}$ in the following way: $D^{+}(\theta)=0$ if $\theta \in T$, and $D^{+}(\theta)=D(\theta)$ otherwise; that is, $D^{+}(W)=D\left(W^{+}\right)$. This degree function provides one more complete order on the set of all words in $P_{T}$. We shall write $W \ll V$ provided that $D(W)<D(V)$, or $D(W)=D(V) \& D^{+}(W)<D^{+}(V)$, or $D(W)=D(V) \& D^{+}(W)=D^{+}(V) \&$ $W<V$. The following lemma shows that this order is compatible with the PBWdecomposition related to $P_{T}$ provided that $U$ is $\operatorname{ad}_{l}$-invariant.

Lemma 5.2. Under the conditions of Theorem 5.1, if $W$ is a word in $P_{T}$, then every monotonous restricted word $V$ in the $P_{T}$-decomposition of $W$ satisfies $D^{+}(V) \leq$ $D^{+}(W)$.

Proof. We shall use induction on words ordered by the Hall order $\prec$. Let $W=\theta_{1} \theta_{2}$. If $\theta_{1}, \theta_{2} \in T$, then the statement follows from Proposition 4.4. If $\theta_{1}, \theta_{2} \notin T$, then $D^{+}(W)=D(W)$, and $D^{+}(V) \leq D(V)=D(W)$. If $\theta_{1}<\theta_{2}$, then $W$ is a basis word and there is nothing to prove. It remains to check the following two cases.

a) $\theta_{1}=[u]>\theta_{2} \in T,[u] \notin T$. By Lemma 3.5 and Lemma 4.5 we have

$$
\operatorname{ad}_{l}([u])(\theta)=[u] \theta+g_{u} \theta S([u])+\sum \alpha_{i} g_{i} W_{i}^{\prime} \theta S\left(W_{i}^{\prime \prime}\right) \in \mathbf{U},
$$


where $W_{i}^{\prime}$ are words in $P_{T}$ starting with less than $[u]$ letters. By means of this formula we have

$$
[u] \theta_{2}=\operatorname{ad}_{l}([u])\left(\theta_{2}\right)-g_{u} \theta_{2} S([u])-\sum \alpha_{i} g_{i} W_{i}^{\prime} \theta_{2} S\left(W_{i}^{\prime \prime}\right) .
$$

The first summand in the right hand side belongs to $\mathbf{U}$. All others start with letters that are less than $[u]$; hence we may apply the inductive supposition. Since $D^{+}\left(\theta_{2} S([u])\right) \leq D(S([u]))=D([u])=D^{+}\left([u] \theta_{2}\right)$, and $D^{+}\left(W_{i}^{\prime} \theta_{2} W_{i}^{\prime \prime}\right) \leq D\left(W_{i}^{\prime} W_{i}^{\prime \prime}\right)$ $=D([u])=D^{+}\left([u] \theta_{2}\right)$, we get the statement.

b) $\theta_{1}>\theta_{2}=[u] \notin T, \theta_{1} \in T$. By definition of the antipode, we have $\operatorname{ad}_{l}([u])(1)=$ $\varepsilon(u)=0$; that is, in terms of (5.1), we get

$$
S([u])=-g_{u}^{-1}[u]-\sum \alpha_{i} g_{u}^{-1} g_{i} W_{i}^{\prime} S\left(W_{i}^{\prime \prime}\right) .
$$

Since $g_{u} \theta_{1} g_{u}^{-1}=p_{\theta_{1}, u}^{-1} \theta_{1}$, the substitution of $S([u])$ in (5.1) implies

$$
\begin{gathered}
\theta_{1}[u]=-p_{\theta_{1}, u} \operatorname{ad}_{l}([u])\left(\theta_{1}\right)+p_{\theta_{1}, u}[u] \theta_{1} \\
-p_{\theta_{1}, u} \sum \alpha_{i} g_{i} W_{i}^{\prime} \theta_{1} S\left(W_{i}^{\prime \prime}\right)-\sum \alpha_{i} \theta_{1} g_{i} W_{i}^{\prime} S\left(W_{i}^{\prime \prime}\right) .
\end{gathered}
$$

One may apply the inductive supposition to the right hand side in the same way as we have done in the case a).

Let $W$ have more than two letters, $W=\theta_{1} \theta_{2} \ldots \theta_{n}, n \geq 3$, and $D^{+}(V)>D^{+}(W)$ for one of the $V$ 's that appears in the $P_{T}$-decomposition of $W$. If $\theta_{2} \ldots \theta_{n}$ is not monotonous and restricted, then by Lemma 4.6 and the inductive supposition it is a linear combination over $\mathbf{k}[G]$ of smaller basis words $V_{i}$ with $D^{+}\left(V_{i}\right) \leq D^{+}\left(\theta_{2} \ldots \theta_{n}\right)$. Since $\theta_{1} V_{i} \prec \theta_{1} \ldots \theta_{n}$, we may use the inductive supposition again. Thus $\theta_{2} \ldots \theta_{n}$ is monotonous and restricted.

In perfect analogy $\theta_{1} \ldots \theta_{n-1}$ is monotonous and restricted too. This implies that $W$ is monotonous. It is not restricted only if $\theta_{1}=\theta_{2}=\ldots=\theta_{n}, n=h_{T}(\theta)$. If $\theta \in T$, then by Proposition 4.4 all $V$ 's are words in $T$; hence $D^{+}(V)=0=D^{+}(W)$. If $\theta \notin T$, then $D^{+}(W)=D(W)$, and $D^{+}(V) \leq D(V)=D(W)$.

Lemma 5.3. If in Lemma 5.2 the word $W^{-}$is monotonous and restricted, then either $D^{+}(V)<D^{+}(W)$, or $V^{-}=W^{-} \& V^{+} \leq W^{+}$.

Proof. Induction on words ordered by $\prec$. Let $W=\theta_{1} \theta_{2}$. In this case $\theta_{1}>\theta_{2}$, and either $\theta_{1}=[u] \notin T, \theta_{2} \in T$, or $\theta_{1} \in T, \theta_{2}=[u] \notin T$ (otherwise there is nothing to prove: if $\theta_{1}, \theta_{2} \notin T$, we have in mind Lemma 4.6).

If $\theta_{1}=[u] \notin T$, then formulae (5.2) and (5.3) with Proposition 4.4. Lemma 4.6. and the inductive supposition imply the required statement.

If $\theta_{2}=[u] \notin T$, then formula (5.4) with the inductive supposition imply the statement.

Let $W$ have more than two letters, $W=\theta_{1} \theta_{2} \cdots \theta_{n}, n \geq 3$, and suppose that one of the basis words in the decomposition of $W$, say $V$, does not fulfill the conclusion of the lemma.

If $\theta_{2} \cdots \theta_{n}$ is not a basis word, then by Lemma 4.6 and the inductive supposition, it is a linear combination over $\mathbf{k}[G]$ of smaller basis words $V_{i}$ with $D^{+}\left(V_{i}\right)<$ $D^{+}\left(\theta_{2} \cdots \theta_{n}\right)$, or $V_{i}^{-}=\left(\theta_{2} \cdots \theta_{n}\right)^{-} \& V_{i}^{+} \leq\left(\theta_{2} \cdots \theta_{n}\right)^{+}$. The word $V$ must appear in the decomposition of one of the words $\theta_{1} V_{i}$. Moreover, since $D^{+}(V)=$ $D^{+}(W)$, the word $V$ may appear only in the decomposition of $\theta_{1} V_{i}$ with $D^{+}\left(V_{i}\right)=$ $D^{+}\left(\theta_{2} \cdots \theta_{n}\right)$ (we have in mind Lemma 5.2). Hence $V_{i}^{-}=\left(\theta_{2} \cdots \theta_{n}\right)^{-}, V_{i}^{+} \leq$ $\left(\theta_{2} \cdots \theta_{n}\right)^{+}$. The inductive supposition applied to $\theta_{1} V_{i} \prec \theta_{1} \theta_{2} \cdots \theta_{n}$ yields $V^{-}=$ 
$\left(\theta_{1} V_{i}\right)^{-}=\theta_{1}^{-} \cdot V_{i}^{-}=\theta_{1}^{-} \cdot\left(\theta_{2} \cdots \theta_{n}\right)^{-}=W^{-}$, and $V^{+} \leq\left(\theta_{1} V_{i}\right)^{+}=\theta_{1}^{+} \cdot V_{i}^{+} \leq$ $\theta_{1}^{+} \cdot\left(\theta_{2} \cdots \theta_{n}\right)^{+}=W^{+}$; that is, $V$ fulfills the lemma conditions. This contradiction shows that $\theta_{2} \cdots \theta_{n}$ is monotonous and restricted.

In the same way $\theta_{1} \cdots \theta_{n-1}$ is monotonous and restricted. Therefore $W$ itself is monotonous. It is not restricted only if $W=\theta^{n}, n=h_{T}(\theta)$. If $\theta \in T$, then $W=W^{-}$ is restricted by the conditions of the lemma. If $\theta=[u] \notin T$, then $W^{+}=W, W^{-}=\emptyset$. Hence by Lemma 4.6 we have $V^{+}<W=W^{+}$, and $D\left(V^{-}\right)=D(V)-D^{+}(V)=0$. The lemma is proved.

Lemma 5.4. Under the conditions of Lemma 5.2, if both $W^{-}$and $W^{+}$are monotonous and restricted, then the leading term with respect to $\ll$ in the $P_{T}$-decomposition of $W$ equals $\alpha W_{1} \circ W_{2}, \alpha \neq 0$.

Proof. We use induction on words ordered by $\ll$. Let $W=\theta_{1} \theta_{2}$. In this case $\theta_{1}>\theta_{2}$, and either $\theta_{1}=[u] \notin T, \theta_{2} \in T$, or $\theta_{1} \in T, \theta_{2}=[u] \notin T$ (otherwise there is nothing to prove).

If $\theta_{1}=[u] \notin T$, then formulae (5.2) and (5.3) with Proposition 4.4 and Lemma 4.6 imply that the leading term with respect to $\ll$ of $[u] \theta_{2}$ equals $p_{\theta_{2}, u}^{-1} \theta_{2}[u]$.

If $\theta_{2}=[u] \notin T$, then formula (5.4) with Proposition 4.4 implies that the leading term with respect to $\ll$ of $\theta_{1}[u]$ equals $p_{\theta_{1}, u}[u] \theta_{1}$.

Suppose that $W=\theta_{1} \ldots \theta_{n}, n \geq 3$. Lemma 5.3 implies that every basis word

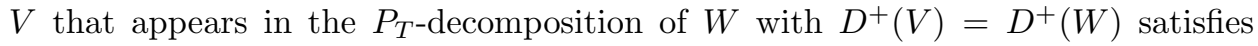
$V^{-}=W^{-}$and $V^{+} \leq W^{+}$. Hence $V \leq W^{-} \circ W^{+}$.

By the inductive supposition the leading term with respect to $\ll$ of $W_{1}=\theta_{2} \ldots \theta_{n}$ equals $\alpha W_{1}^{-} \circ W_{1}^{+}, \alpha \neq 0$; that is, $W_{1}-\alpha W_{1}^{-} \circ W_{1}^{+}=\sum \beta_{i} g_{i} V_{i}$, where $V_{i} \ll$ $W_{1}^{-} \circ W_{1}^{+}$are basis words. By Lemma 5.3 this implies $V_{i}^{-}=W_{1}^{-}$, and $V_{i}^{+}<W_{1}^{+}$ provided that $D^{+}\left(V_{i}\right)=D^{+}\left(W_{1}\right)$. In particular $\left(\theta_{1} V_{i}\right)^{+}<\theta_{1} W_{1}^{+}=W^{+}$; that is, again by Lemma 5.3. the word $W^{-} \circ W^{+}$does not appear in the decomposition of $\theta_{1} V_{i}$, but it may appear in the decomposition of $\alpha \theta_{1}\left(W_{1}^{-} \circ W_{1}^{+}\right)$. Hence it remains to prove the statement for the word $\theta_{1}\left(W_{1}^{-} \circ W_{1}^{+}\right)$, or, equivalently, we may suppose that $\theta_{2} \ldots \theta_{n}$ is monotonous and restricted.

We consider in that setting the word $W_{2}=\theta_{1} \ldots \theta_{n-1}$. By the above arguments, applying the inductive supposition to $W_{2}$, we see that the word $W^{-} \circ W^{+}$may appear only in the decomposition of $\beta\left(W_{2}^{-} \circ W_{2}^{+}\right) \theta_{n}, \beta \neq 0$. The word $\left(W_{2}^{-} \circ W_{2}^{+}\right) \theta_{n}$ is not equal to $\left(W^{-} \circ W^{+}\right)$only if $\theta_{1}$ is the biggest letter of $W$. If so, then the letter $\theta_{n}$ is not the smallest letter of the word $\left(W_{2}^{-} \circ W_{2}^{+}\right) \theta_{n} \stackrel{d f}{=} \theta_{1}^{\prime} \theta_{2}^{\prime} \ldots \theta_{n-1}^{\prime} \theta_{n}$, and $\theta_{1}^{\prime} \theta_{2}^{\prime} \ldots \theta_{n-1}^{\prime}=W_{2}^{-} \circ W_{2}^{+}$is monotonous and restricted. Applying for the third time the inductive supposition to $W_{3}=\theta_{2}^{\prime} \ldots \theta_{n-1}^{\prime} \theta_{n}$, we have that $W^{-} \circ W^{+}$may appear (and does appear) only in the decomposition of $\gamma \theta_{1}^{\prime}\left(W_{3}^{-} \circ W_{3}^{+}\right)=\gamma W^{-} \circ W^{+}$, $\gamma \neq 0$.

Proof of Theorem [5.1. Consider a linear map $\varphi: \mathbf{U} \otimes_{\mathbf{k}} H^{+} \rightarrow H$ given by $\varphi\left(g W_{1} \otimes W_{2}\right)=g W_{1} \cdot W_{2}$, where $\cdot$ is the multiplication in $H$, while $W_{1}$ runs through the set of all monotonous restricted words in $T$, and $W_{2}$ runs through the set of all monotonous restricted words in $P_{T} \backslash T, g \in G$. Certainly this map is a homomorphism of left $\mathbf{U}$-modules. We need to prove that $\varphi$ is an isomorphism.

We shall check by induction with respect to $\ll$ that the value of every monotonous restricted word $W$ in $P_{T}$ is a linear combination of the products $g W_{1} W_{2}$. According to Lemma 5.4 the leading term with respect to $\ll$ in the $P_{T}$-decomposition 
of $W^{-} W^{+}$equals $\alpha W^{-} \circ W^{+}=\alpha W, \alpha \neq 0$. It remains to apply the inductive supposition to $W-\alpha^{-1} W^{-} W^{+}$. Hence $\operatorname{im} \varphi=H$.

Let $\sum \alpha_{i} g_{i} W_{1}^{i} W_{2}^{i}=0$ in $H$. Denote by $W$ the maximal word with respect to $\ll$ among $W_{1}^{i} \circ W_{2}^{i}$. Lemma 5.4 implies that the leading term with respect to $\ll$ of $\sum \alpha_{i} g_{i} W_{1}^{i} W_{2}^{i}$ in the $P_{T}$-decomposition equals $\left(\sum \alpha \alpha_{i} g_{i}\right) W, \alpha \neq 0$, where $i$ runs through the set $J$ of all indices with $W_{1}^{i}=W^{-}, W_{2}^{i}=W^{+}$. Since the correspondence $W \rightarrow\left(W^{-}, W^{+}\right)$is bijective, all $g_{i}$ in the latter sum are different. Hence $\left(\sum \alpha \alpha_{i} g_{i}\right) W \neq 0$. Thus $\operatorname{ker} \varphi=0$.

In order to prove that $H$ is a free right module over $\mathbf{U}$, we consider a map $\psi: H^{+} \otimes_{\mathbf{k}} \mathbf{U} \rightarrow H$ given by $\psi\left(W_{2} \otimes g W_{1}\right)=W_{2} g W_{1}=\lambda g W_{2} \cdot W_{1}, \lambda \neq 0$, where as above $W_{1}, W_{2}$ run through the sets of all monotonous restricted words respectively in $T$ and in $P_{T} \backslash T, g \in G$. This map is a homomorphism of right $\mathbf{U}$-modules. By Lemma 5.4, the leading term with respect to $\ll$ of $W^{+} W^{-}$equals $\alpha W, \alpha \neq 0$, where $W$ is an arbitrary monotonous restricted word in $P_{T}$. This allows us to replace $\varphi$ with $\psi$ in the above arguments. Hence $\psi$ also is an isomorphism. Theorem 5.1 is proved.

Remark. We have to note that if $U$ is not $\operatorname{ad}_{l}$-invariant, then neither Lemma 5.2 nor Lemma 5.4 are valid. Consider, for example, the free algebra $H=\mathbf{k}\langle x, y\rangle$ with $\mathbf{U}=\mathbf{k}\langle x\rangle$, and suppose $x>y$. Then $x \cdot y=[x y]+p_{x y} y \cdot x$ is the $P_{T^{-}}$-decomposition, while its leading term equals $[x y]$ with $D^{+}([x y])=D(x)+D(y)>D(y)=D^{+}(x \cdot y)$.

\section{An application to pointed Hopf algebras}

If the ground field is algebraically closed, the main results admit a nice generalization inspired by [43, Theorem 47]. We are reminded that the space $P$ spanned by all skew primitive elements in any pointed Hopf algebra is a Yetter-Drinfeld module over the coradical $\mathbf{k}[F]$; that is, $P$ is graded by the group $F$ of all group-like elements and $F$ acts on $P$ so that

$$
P=\bigoplus_{g \in F} P_{g}, \quad P_{g}^{h}=P_{h^{-1} g h}, \quad g, h \in F,
$$

where $P_{g}=\{a \in H: \Delta(a)=a \otimes 1+g \otimes a\}$.

Theorem 6.1. Suppose that the field $\mathbf{k}$ is algebraically closed. Let a Hopf algebra $H$ be generated by a commutative group $G$ of group-like elements and a subspace $V=\bigoplus_{k \in I} V_{k}, V_{k} \subseteq P_{g(k)}, g(k) \in G$. If $\operatorname{dim}_{\mathbf{k}} V_{k}<\infty$ and $V_{k}^{g} \subseteq V_{k}$ for all $k \in I$, $g \in G$, then every right coideal subalgebra $\mathbf{U} \supseteq \mathbf{k}[G]$ has a $P B W$-basis over $\mathbf{k}[G]$ that may be extended up to a PBW-basis of $H$ over $\mathbf{k}[G]$.

The statement follows from Lemma 2.3 and Theorem 4.1 by means of the proposition below.

Proposition 6.2. Under the conditions of Theorem 6.1 there exists a filtration on $H$ such that the associated graded Hopf algebra is a character Hopf algebra.

Proof. Recall that in the context of Theorem 6.1 every irreducible finite-dimensional representation of an Abelian group is one-dimensional. Hence each of the $V_{k}$ 's has a flag of $G$-invariant subspaces

$$
(0) \subset \mathbf{k} x_{1, k} \subset \mathbf{k} x_{1, k}+\mathbf{k} x_{2, k} \subset \ldots \subset \mathbf{k} x_{1, k}+\mathbf{k} x_{2, k}+\cdots+\mathbf{k} x_{n, k}=V_{k} .
$$

Let us fix an arbitrary complete order on the set $G$. The set $X=\bigcup_{k \in I}\left\{x_{i, k} \mid i \in \mathbf{Z}\right\}$ ordered by $x_{i, k}<x_{j, h} \Longleftrightarrow k<h$ or $k=h \& i<j$ is a completely ordered basis of 
$V$. Consider the filtration given in Example 2.2 with $A=\mathbf{k}[G]$. Since all elements $x_{i, k}$ are primitive, this is a Hopf algebra filtration. Definition (2.4) implies that $\operatorname{gr} H$ is generated by $\bar{X}=\operatorname{gr} X$ and $\operatorname{gr} \mathbf{k}[G]=\mathbf{k}[G]$. It remains to note that all $\bar{x}$, $x \in X$ are semi-invariants:

$$
x_{i, k}^{h}=\alpha_{h, i} x_{i, k}+\beta_{1} x_{i-1, k}+\ldots+\beta_{i-1} x_{1, k} \equiv \alpha_{h, i} x_{i, k} \quad\left(\bmod H_{\gamma}^{-}\right),
$$

where according to Eq. (2.3) we have $\gamma \stackrel{d f}{=} D\left(x_{i, k}^{h}\right)=x_{i, k} \in \Gamma$. The proposition is proved.

In perfect analogy, Theorem 5.1 with Lemma 2.4 imply:

Corollary 6.3. If in Theorem 6.1 the right coideal subalgebra $\mathbf{U}$ is a bosonization of an $\mathrm{ad}_{l}$-invariant subalgebra $U$, then $H$ has a free left and right $P B W$-basis over U.

\section{BRAIDED VERSION}

The main results can be restated in terms of braided Hopf algebras with diagonal braidings. More precisely these are Hopf algebras in a braided tensor category of bigraded spaces, spaces graded by $G \times G^{*}$, where $G$ is a fixed Abelian group, and $G^{*}$ is the group of its characters; see [41] for the axioms of braided Hopf algebras.

Corollary 7.1. Let $S$ be a Hopf algebra in the braided tensor category of bigraded spaces. If $S$ is generated by a bigraded subspace of primitive elements, then each bigraded right coideal subalgebra $U$ has a $P B W$-basis that may be extended up to a $P B W$-basis of $R$. If $U$ is $\operatorname{ad}_{l}$-invariant, then $S$ has a $P B W$-basis over $U$, and it is left and right free over $U$.

Proof. Let $S=\bigoplus_{g \in G, \chi \in G^{*}} S_{g}^{\chi}$ be the bigrading. The group algebra acts on $S$ via $s^{g}=\chi(g) s$, where $s \in S_{\bullet}^{\chi}$. This action defines the skew group algebra $H=\mathbf{k}[G] * R$ that gets a structure of a character Hopf algebra if we put (this is a particular case of the Radford biproduct [30])

$$
\Delta(x)=x \otimes 1+g \otimes x, \Delta(g)=g \otimes g, \quad x \in S_{g}^{\bullet} .
$$

In this case the coproduct $\Delta$ and antipode $\sigma$ of $H$ are related with the coproduct $\Delta^{b}$ and antipode $\sigma^{b}$ of $S$ in the following way:

$$
h_{(1)}=h_{(1)}^{b} g_{2}, \quad h_{(2)}=h_{(2)}^{b}, \quad \sigma(h)=g_{h}^{-1} \sigma^{b}(h),
$$

where $h^{b} \in S_{g_{h}}^{\bullet}, h_{(2)}^{b} \in S_{g_{2}}^{\bullet}$. Hence $U$ is an ad $_{l}$-invariant subalgebra in $H$, provided that it is so in $S$, and we may apply Theorem 4.1 and Theorem 5.1 .

Lemmas 2.3 and 2.4 allow us to generalize this statement as follows. Let $V$ be a linear space with an invertible braiding $\tau: V \otimes V \rightarrow V \otimes V$. Recall that a subspace $W$ of $V$ is called right categorical if $\tau(V \otimes W) \subseteq W \otimes V$. It is left categorical if $\tau(W \otimes V) \subseteq V \otimes W$. A left and right categorical subspace is categorical.

Suppose that $V$ has a completely ordered basis $X$. For each $x \in X$ we denote by $V_{\leq x}$ a subspace spanned by all $y \leq x$, respectively $V_{<x}=\sum_{y<x} V_{\leq y}$. We shall call the basis $X$ categorically ordered if all such spaces are categorical:

$$
\tau\left(V_{\leq x} \otimes V_{\leq y}\right) \subseteq V_{\leq y} \otimes V_{\leq x} .
$$


Theorem 7.2. Every right coideal subalgebra $U$ of a braided Hopf algebra $R$ generated by a braided subspace of primitive elements with categorically ordered basis has a set of $P B W$-generators that can be extended up to a set of PBW-generators of $R$. If $U$ is $\operatorname{ad}_{l}$-invariant, then $R$ has a $P B W$-basis over $U$, and it is left and right free over $U$.

Due to Lemmas 2.3 2.4 we need just the following proposition.

Proposition 7.3. Under the conditions of Theorem 7.2 there exists a filtration on $R$ such that the associated graded algebra satisfies the conditions of Corollary 7.1,

Proof. The filtration given in Example 2.2 with $A=\mathbf{k}$ is the required one. To see this we have to check the following relations:

$$
\begin{array}{ll}
\text { 1. } & \tau\left(R_{\gamma} \otimes R_{\delta}\right) \subseteq R_{\delta} \otimes R_{\gamma}, \quad \delta, \gamma \in \Gamma ; \\
\text { 2. } & \Delta^{b}\left(R_{\gamma}\right) \subseteq \sum_{\delta+\varepsilon=\gamma} R_{\delta} \otimes R_{\varepsilon} \delta, \varepsilon, \gamma \in \Gamma ; \\
\text { 3. } & \sigma^{b}\left(R_{\gamma}\right) \subseteq R_{\gamma}, \quad \gamma \in \Gamma ; \\
\text { 4. } & \tau(x \otimes y)-p_{x, y} y \otimes x \in R_{<y} \otimes R_{\leq x}+R_{\leq y} \otimes R_{<x}, p_{x, y} \neq 0 .
\end{array}
$$

Since the map $\xi: \mathbf{k}\langle X\rangle \rightarrow R$ is a homomorphism of braided Hopf algebras, and $\xi\left(\mathbf{k}\langle X\rangle_{\gamma}\right)=R_{\gamma}$, see (2.4), it suffices to verify these relations in the free algebra $\mathbf{k}\langle X\rangle$.

1. We shall use the explicit form for the extension of $\tau$ on $\mathbf{k}\langle X\rangle$ given in [15, Eq. (17)]:

$$
\tau(u \otimes t v)=\sum_{s \in X} s \tau\left(A_{t}^{s}(u) \otimes v\right), \quad t \in X,
$$

where $A_{t}^{s}: \mathbf{k}\langle X\rangle \rightarrow \mathbf{k}\langle X\rangle, t, s \in X$ are linear maps uniquely defined by the relations

$$
A_{t}^{s}(u v)=\sum_{j \in X} A_{j}^{s}(u) A_{t}^{j}(v), \quad s, t \in X,
$$

and by the initial conditions $\tau(x \otimes y)=\sum_{s \in X} s \otimes A_{y}^{s}(x), x, y \in X$.

The definition of categorical ordering (7.1) implies $A_{t}^{s}(x)=0, s>t, x \in X$. By means of (7.7) and evident induction we have $A_{t}^{s}(u)=0, s>t, u \in \mathbf{k}\langle X\rangle$.

Suppose that $\mathbf{k}\langle X\rangle_{\delta}, \delta<\gamma$ are right categorical. Let $w$ be a word of degree $\gamma$, $w=t v, t \in X$. Then $D(v)<D(w)$. Equation (7.6) implies

$$
\tau(\mathbf{k}\langle X\rangle \otimes t v) \subseteq \sum_{s \leq t} s \mathbf{k}\langle X\rangle_{D(v)} \otimes \mathbf{k}\langle X\rangle \subseteq \mathbf{k}\langle X\rangle_{\gamma} \otimes \mathbf{k}\langle X\rangle
$$

since $s+D(v) \leq t+D(v)=\gamma$. Thus $\mathbf{k}\langle X\rangle_{\gamma}$ is right categorical.

The definition of categorical ordering is left-right symmetric; thus $\mathbf{k}\langle X\rangle_{\gamma}$ is left categorical as well; that is, (7.2) is fulfilled.

2. We are reminded that the braided coproduct is a homomorphism $\Delta^{b}: R \rightarrow R \otimes R$, where $R \underline{\otimes} R$ is the ordinary tensor product of linear spaces with a new multiplication

$$
(a \underline{\otimes} b)(r \underline{\otimes} v)=a \tau(b \underline{\otimes} r) v .
$$

This definition and Eq. (7.2) imply

$$
\left(R_{\gamma} \underline{\otimes} R_{\delta}\right)\left(R_{\varepsilon} \underline{\otimes} R_{\mu}\right) \subseteq\left(R_{\gamma} R_{\varepsilon} \underline{\otimes} R_{\delta} R_{\mu}\right) .
$$

Since all generators are primitive, we get (7.3).

3. The antipode $\sigma^{b}$ is an antihomomorphism in the braided category. Since $\sigma^{b}(x)=$ $-x, x \in X$, formula (7.2) implies (7.4) by an evident induction. 
4. We just need to show that $p_{x, y} \neq 0$. Let us prove simultaneously the equalities

$$
\tau^{-1}\left(V_{\leq y} \otimes V_{\leq x}\right)=V_{\leq x} \otimes V_{\leq y}
$$

If $x$ is the smallest element from $X$, then (7.1) implies $\tau(x \otimes x)=p_{x, x} x \otimes x$. Hence $\tau^{-1}(x \otimes x)=p_{x, x}^{-1} x \otimes x$, and $p_{x, x} \neq 0$.

In the general case we use induction on $D(x)+D(y)=x+y \in \Gamma$. We have

$$
\tau(x \otimes y)-p_{x, y} y \otimes x \in V_{<y} \otimes V_{\leq x}+V_{\leq y} \otimes V_{<x} .
$$

If $p_{x, y}=0$, then we arrive at a contradiction,

$$
x \otimes y \in \tau^{-1}\left(V_{<y} \otimes V_{\leq x}+V_{\leq y} \otimes V_{<x}\right)=V_{\leq x} \otimes V_{<y}+V_{<x} \otimes V_{\leq y},
$$

where we have used the inductive supposition (7.9). Hence $p_{x, y} \neq 0$, and

$$
\tau^{-1}\left(V_{\leq y} \otimes V_{\leq x}\right)=\mathbf{k}(x \otimes y)+\tau^{-1}\left(V_{<x} \otimes V_{\leq y}+V_{\leq x} \otimes V_{<y}\right)=V_{\leq x} \otimes V_{\leq y}
$$

Thus $\operatorname{gr} R$ is a braided Hopf algebra generated by a subspace $\operatorname{gr} V=V$ of primitive elements with diagonal braiding, $\tau(x \otimes y)=p_{x, y} y \otimes x$. One may define bigrading on $\operatorname{gr} R$ as follows. Let $G$ be a free Abelian group freely generated by elements $g_{i}$, $i \in I$. Denote by $\chi^{i}$ the character $\chi^{i}\left(g_{j}\right)=p_{x_{j}, x_{i}}^{-1}$. We let $x_{i} \in R_{g_{i}}^{\chi^{i}}$ and extend the bigrading multiplicatively. Since gr $R$ and gr $U$ are homogeneous in each of the generators $x_{i}$, they are bigraded spaces as well.

The braided Hopf algebras generated by ordered sets of primitive elements have been considered by S. Ufer in the above cited paper [43. He has proved that every braided Hopf algebra generated by a braided subspace $V$ spanned by a completely ordered set $X$ of primitive elements with the left triangular braiding,

$$
\tau(x \otimes y)-p_{x, y} y \otimes x \in V_{<y} \otimes V, \quad 0 \neq p_{x, y} \in \mathbf{k}, x, y \in X,
$$

has a PBW-basis [43, Theorem 34]. Formula (7.10) certainly implies that subspaces $V_{\leq y}$ are right categorical, but in general they are not left categorical. At the same time if $X$ is categorically ordered, then the braiding is not necessarily left triangular. We remark that $\mathrm{S}$. Ufer considers $X$ to be finite. Nevertheless he uses this assumption just to produce the (downward) induction (see [43, Definition 32] and the comments after that); hence, in fact, his result covers the case of infinite $X$ as well. To avoid confusion we should stress that the order on $X$ that we discuss here is opposite to that considered in [43].

\section{REFERENCES}

1. N. Andruskiewitsch and H.-J. Schneider, Pointed Hopf algebras, in: S. Montgomery, H.-J. Schneider (Eds.), New Directions in Hopf Algebras, MSRI Publications, 43(2002), 1-68(1998), 3297-3319. MR1913436 (2003e:16043)

2. G. Benkart, S.-J. Kang, and D. Melville, Quantized enveloping algebras for Borcherds superalgebras, Trans. Amer. Math. Soc., 350,N8(1998), 3297-3319. MR1451594 (99f:17014)

3. V. Chari and N. Xi, Monomial basis of quantized enveloping algebras, in: Recent development in quantum affine algebras and related topics, 248, AMS, Contemp. Math., 1999, pp. 69-81. MR:1745255 (2001c:17023)

4. M. Costantini and M. Varagnolo, Multiparameter quantum function algebra at roots of 1 , Math. Ann. 306, N4(1996), 759-780. MR1418352 (97i:17012)

5. B. Deng and J. Du, Bases of quantized enveloping algebras, Pacific Journal of Math., v. 220, N1(2005), 33-48. MR2195061 (2006j:17015)

6. B. Deng and J. Du, Monomial bases for quantum $s_{n}$, Advances in Mathematics, vol. 191, N2(2005), 276-304. MR2103214 (2005i:17015) 
7. V.O. Ferreira, L.S.I. Murakami, and A. Paques, A Hopf-Galois correspondence for free algebras, J. Algebra, 276(2004), 407-416. MR2054404(2005a:16054)

8. D. Flores and J.A. Green, Quantum symmetric algebras, Algebras and Representation Theory, 4, N1(2001), 55-76. MR1825807 (2002f:17021)

9. F. Gavarini, A PBW basis for Lusztig's form of untwisted affine quantum groups, Comm. in Algebra, 27(2)(1999), 903-918. MR1672011 (2000b:17016)

10. M. Graña, A freeness theorem for Nichols algebras, J. Algebra, 231(2000), 235-257. MR 1779599 (2001g:16077)

11. S.-J. Kang, Quantum deformations of generalized Kac-Moody algebras and their modules, Journal of Algebra, 175(1995), 1041-1066. MR1341758 (96k:17023)

12. V.K. Kharchenko, A quantum analog of the Poincaré-Birkhoff-Witt theorem, Algebra i Logika, 38, N4(1999), 476-507. English translation: Algebra and Logic, 38, N4(1999), 259-276 (QA/0005101). MR:1763385 (2001f:16075)

13. V.K. Kharchenko, Skew primitive elements in Hopf algebras and related identities, Journal of Algebra, 238(2001), 534-559. MR1823773 (2002b:16055)

14. V.K. Kharchenko, A combinatorial approach to the quantifications of Lie algebras, Pacific Journal of Mathematics, 203, N1(2002), 191-233. MR1895931(2003b:17018)

15. V.K. Kharchenko, Constants of coordinate differential calculi defined by Yang-Baxter operators, Journal of Algebra, 267, N1(2003), 96-129. MR1993469 (2004e:16043)

16. B. Leclerc, Dual canonical bases, quantum shuffles and q-characters, Math. Z. 246, N4(2004), 691-732. MR2045836 (2005c:17019)

17. G. Letzter, Coideal subalgebras and quantum symmetric pairs, in: S. Montgomery, H.-J. Schneider (Eds.) New Directions in Hopf Algebras, MSRI Publications, 43(2002), 117-165. MR 1913438 (2003g:17025)

18. M. Lothaire, Combinatorics on Words, Encyclopedia of Mathematics and its Applications, Addison-Wesley Publ. Co., 1983. MR675953 (84g:05002)

19. G. Lusztig, Finite-dimensional Hopf algebras arising from quantized enveloping algebras, J. Amer. Math. Soc. 3(1)(1990) 257-296. MR1013053(91e:17009)

20. G. Lusztig, Introduction to Quantum Groups, Progress in Mathematics 110, BirkhäuserBoston, 1993. MR 1227098 (94m:17016)

21. A. Masuoka, Freeness of Hopf algebras over coideal subalgebras, Comm. in Algebra, 20, N5(1992), 1353-1373. MR1157912 (93d:16051)

22. S. Montgomery, Hopf Algebras and Their Actions on Rings, CBMS, 82, AMS, Providence, 1993. MR.1243637 (94i:16019)

23. W.D. Nichols and M.B. Zoeller, A Hopf algebra freeness theorem, Amer. J. Math., 111(1989), 381-385. MR.987762 (90c:16008)

24. W.D. Nichols and M.B. Zoeller, Freeness of infinite-dimensional Hopf algebras over grouplike subalgebras, Comm. Algebra 17, N2(1989), 413-424. MR978483 (90c:16009)

25. W.D. Nichols and M.B. Richmond (Zoeller), Freeness of infinite-dimensional Hopf algebras, Comm. Algebra, 20(1992), 1489-1492. MR1157919 (93d:16054)

26. U. Olberst and H.-J. Schneider, Untergruppen formeller Gruppen von endlichen Index, Journal of Algebra, 31(1974), 10-44. MR0360610 (50:13057)

27. D.E. Radford, Pointed Hopf algebras are free over Hopf subalgebras, J. Algebra, 45, N2(1977), 266-273. MR0437582 (55:10506)

28. D.E. Radford, Freeness (projectivity) criteria for Hopf algebras over Hopf subalgebras, J. Pure Appl. Algebra 11(1977), 15-28. MR0476790 (57:16344)

29. D.E. Radford, On an analog of Lagrange's theorem for commutative Hopf algebras, Proc. Amer. Math. Soc. 79, N. 2(1980), 164-166. MR565330 (81c:16011)

30. D.E. Radford, Hopf algebras with projection, Journal of Algebra, 92(1985), 322-347. MR 778452 (86k:16004)

31. M. Reineke, Feigin's map and monomial bases for quantized enveloping algebras, Math. Z. 237(3)(2001) 639-667. MR1845343(2002f:17029)

32. C.M. Ringel, PBW-bases of quantum groups, J. Reine Angew. Math. 470(1996), 51-88. MR:1370206 (97d:17009)

33. M. Rosso, An analogue of the Poincaré-Birkhoff-Witt theorem and the universal R-matrix of $U_{q}(\operatorname{sl}(N+1))$, Comm. Math. Phys., vol. 124(1989), 307-318.

34. B. Scharfschwerdt, The Nichols-Zoeller theorem for Hopf algebras in the category of YetterDrinfeld modules, Comm. Algebra 29, N6(2001), 2481-2487. MR1845124 (2002e:16064) 
35. H.-J. Schneider, Some remarks on exact sequences of quantum groups, Comm. Algebra, 21, N9(1993), 3337-3357. MR.1228767 (94e:17026)

36. A.I. Shirshov, On free Lie rings, Mat. Sb. 45, 87(2)(1958), 113-122. MR0099356 (20:5796)

37. A.I. Shirshov, Some algorithmic problems for Lie algebras, Sibirskii Math. J., 3(2)(1962), 292-296. MR0183753 (32:1231)

38. S. Skryabin, Projectivity and freeness over comodule algebras, Trans. Amer. Math. Soc. 359, N6(2007), 2597-2623. MR.2286047

39. M. Takeuchi, Relative Hopf modules equivalence and freeness criteria, J. Algebra, 60, N2(1979), 452-471. MR549940 (82m:16006)

40. M. Takeuchi, The Nichols-Zoeller theorem for braided Hopf algebras, preprint.

41. M. Takeuchi, Survey of braided Hopf algebras, in: New Trends in Hopf Algebra Theory, Contemp. Math., vol. 267, AMS, Providence, RI, 2000, pp. 301-324. MR.1800719|(2002f:16091)

42. J. Towber, Multiparameter quantum forms of the enveloping algebra $U_{g l_{n}}$ related to the Faddeev-Reshetikhin-Takhtajan $U(R)$ constructions, J. Knot Theory Ramifications 4(5)(1995) 263-317. MR1331751 (96h:17024)

43. S. Ufer, $P B W$ bases for a class of braided Hopf algebras, Journal of Algebra, 280, N1(2004), 84-119. MR2081922 (2005g:16079)

44. H. Yamane, A Poincaré-Birkhoff-Witt theorem for quantized universal enveloping algebras of type $A_{N}$, Publ. RIMS. Kyoto Univ., 25 (1989), 503-520. MR1018513 (91a:17016)

45. T. Yanai, Galois correspondence theorem for Hopf algebra actions, in: Algebraic structures and their representations, 393-411, Contemporary Mathematics, vol. 376, AMS, Providence, RI, 2005. MR2147038(2006c:16072)

FeS-Cuautitlan, Universidad Nacional Autónoma de México, Centro de Investigaciones Teóricas, Primero de Mayo s/n, Campo 1, Cit, Cuautitlan Izcalli, Edstado de MÉxico, 54768, Mexico

E-mail address: vlad@servidor.unam.mx 